\title{
Banach-valued axiomatic spectra
}

\author{
by \\ SeÁn Dineen and Robin E. Harte (Dublin)
}

\begin{abstract}
Using axiomatic joint spectra we obtain a functional calculus which extends our previous Gelfand-Waelbroeck type results to include a Banach-valued TaylorWaelbroeck spectrum.
\end{abstract}

1. Introduction. In previous papers [5-8] we developed a spectral theory and functional calculus for an infinite number of elements in a unital Banach algebra $\mathcal{A}$. In [8] we obtained a functional calculus for the Banachvalued spectrum introduced by Waelbroeck [20] when $\mathcal{A}$ is commutative. In this paper we discuss axiomatic spectra and obtain a holomorphic functional calculus for commuting elements in $\mathcal{A} \widehat{\otimes}_{\gamma} X$ with respect to a class of spectra which includes the commutative case and the Taylor spectrum. The functional calculus is more inclusive than that considered in $[17,22]$ as we consider here a more general class of holomorphic functions.

We refer to Curto [2] for a comprehensive survey of spectral theory for a finite number of commuting elements in a Banach algebra. Background information on tensor products may be found in $[3,16]$ and we refer to $[4,12]$ for information on infinite-dimensional holomorphy.

Throughout this article $\mathcal{A}$ will denote a Banach algebra over the complex numbers $\mathbb{C}$, with identity e. We let $\mathfrak{C}(\mathcal{A})$ and $\mathfrak{C}_{0}(\mathcal{A})$ denote, respectively, the set of all commuting systems of elements and finite commuting systems of elements in $\mathcal{A}$. We let $\mathcal{S}(\mathcal{A})$ denote the spectrum of the unital Banach algebra $\mathcal{A}$, that is, the set of all non-zero multiplicative linear functionals on $\mathcal{A}$. We denote Banach spaces, always assumed to be over $\mathbb{C}$, by $X, Y, \ldots$ and let $\mathcal{B}(X)$ denote the Banach algebra of bounded linear operators from $X$ into $X$.

2. Spectral systems. Axiomatic joint spectra were initially introduced by Słodkowski and Żelazko in 1974 [17]. Since then they have proved useful

2000 Mathematics Subject Classification: 46G25, 46B15, 47A10, 46G20.

Key words and phrases: functional calculus, Taylor-Waelbroeck spectrum, spectral system, polynomial extension. 
both as a theoretical tool and as a means of comparing the different concrete spectra that have been introduced over the years. Slight variations in the definition have also appeared in the literature (see for instance $[2,9,15$, $17,22]$ ), but the differences are generally only a matter of convenience. We have chosen a definition which displays important features but which is not without redundancies and we address this point in the remarks below.

Definition 1 ([2, 17, 22]). A spectral system for the unital Banach algebra $\mathcal{A}$ is a mapping $\sigma^{*}$ which maps $\left(a_{i}\right)_{i \in I} \in \mathfrak{C}_{0}(\mathcal{A})$ onto a compact subset of $\mathbb{C}^{I}$, the spectrum of $\left(a_{i}\right)_{i \in I}$, such that the following hold:

(a) $\sigma^{*}(\{a\})$ is a non-empty subset of $\sigma(a)$, the usual spectrum of $a \in \mathcal{A}$, for any singleton set $\{a\}$ in $\mathfrak{C}_{0}(\mathcal{A})$,

(b) (the finite polynomial spectral mapping property)

$$
P\left(\sigma^{*}(\mathbf{a})\right)=\sigma^{*}(P(\mathbf{a}))
$$

for all $\mathbf{a} \in \mathfrak{C}_{0}(\mathcal{A})$ and all polynomials $P$ between finite-dimensional spaces.

(c) (the projection property)

$$
\pi_{J}^{I}\left(\sigma^{*}\left(\left(a_{i}\right)_{i \in I}\right)\right)=\sigma^{*}\left(\left(a_{i}\right)_{i \in J}\right)
$$

for any subset $J$ of the finite set $I$ where $\pi_{J}^{I}$ denotes the canonical projection from $\mathbb{C}^{I}$ onto $\mathbb{C}^{J}$.

Remarks. (1) Some authors [2, 15] replace (a) with the condition $\sigma^{*}(\{a\})=\sigma(a)$. In either case we have $\sigma^{*}(\{0\})=\sigma(0)=\{0\}$.

(2) Let $\mathbf{a}=\left(a_{1}, \ldots, a_{n}\right) \in \mathfrak{C}_{0}(\mathcal{A})$. If $j=\left(j_{1}, \ldots, j_{n}\right)$ where each $j_{i}$ is a non-negative integer and $z=\left(z_{1}, \ldots, z_{n}\right) \in \mathbb{C}^{n}$, let $z^{j}=z_{1}^{j_{1}} \cdots z_{n}^{j_{n}}$ and $\mathbf{a}^{j}=a_{1}^{j_{1}} \cdots a_{n}^{j_{n}}$. If $P: \mathbb{C}^{n} \rightarrow \mathbb{C}^{m}, P=\left(P_{1}, \ldots, P_{m}\right)$ where each $P_{i}$ is a $\mathbb{C}$-valued polynomial in $n$ variables then each $P_{i}$ is a finite sum $\sum_{J} \alpha_{j} z^{j}$, where $\alpha_{j} \in \mathbb{C}$, and $P_{i}(\mathbf{a})=\sum_{J} \alpha_{j} \mathbf{a}^{j}$.

(3) If $I$ is a finite set and $J \subset I$ then the projection $\pi_{J}^{I}\left(\left(z_{i}\right)_{i \in I}\right):=\left(z_{j}\right)_{j \in J}$ is a polynomial between finite-dimensional spaces, and applying the finite polynomial spectral mapping property we see that $(b) \Rightarrow(c)$ in Definition 1. On the other hand, suppose (a) and (c) hold and also the one way spectral mapping property, that is,

$$
P\left(\sigma^{*}(\mathbf{a})\right) \subset \sigma^{*}(P(\mathbf{a}))
$$

for all $\mathbf{a} \in \mathfrak{C}_{0}(\mathcal{A})$ and all polynomials $P$ between finite-dimensional spaces. Suppose $\mathbf{a}=\left(a_{1}, \ldots, a_{n}\right) \in \mathfrak{C}_{0}(\mathcal{A}), P: \mathbb{C}^{n} \rightarrow \mathbb{C}^{m}$ is a polynomial and $\mu:=\left(\mu_{j}\right)_{j=1}^{m} \in \sigma^{*}(P(\mathbf{a}))$. By the projection property $\mu \in \pi_{m}\left(\sigma^{*}(\mathbf{a}, P(\mathbf{a}))\right)$ where

$$
\pi_{m}\left(z_{1}, \ldots, z_{n}, w_{1}, \ldots, w_{m}\right)=\left(w_{1}, \ldots, w_{m}\right) .
$$


Hence there exists $\lambda=\left(\lambda_{i}\right)_{i=1}^{n} \in \mathbb{C}^{n}$ such that $\left.(\lambda, \mu)=\left(\left(\lambda_{i}\right)_{i=1}^{n}\right),\left(\mu_{j}\right)_{j=1}^{m}\right) \in$ $\sigma^{*}(\mathbf{a}, P(\mathbf{a}))$. By the projection property $\lambda \in \sigma^{*}(\mathbf{a})$. Let

$$
Q\left(z_{1}, \ldots, z_{n}, w_{1}, \ldots, w_{m}\right)=P\left(z_{1}, \ldots, z_{n}\right)-\left(w_{1}, \ldots, w_{m}\right) .
$$

Then $Q$ is a polynomial between finite-dimensional spaces and $Q(\mathbf{a}, \mathbf{b})=$ $P(\mathbf{a})-\mathbf{b}$ for any $\mathbf{b}=\left(b_{j}\right)_{j=1}^{m}$. By the one way polynomial spectral mapping property

$$
Q\left(\sigma^{*}(\mathbf{a}, P(\mathbf{a}))\right) \subset \sigma^{*}(Q(\mathbf{a}, P(\mathbf{a})))=\sigma^{*}(\{\mathbf{0}\})
$$

where $\mathbf{0}=(0, \ldots, 0) \in \mathfrak{C}_{0}(\mathcal{A})$. By the projection property and $(1), \sigma^{*}(\{\mathbf{0}\})=$ $0 \in \mathbb{C}^{n}$. Hence $Q(\lambda, \mu)=P(\lambda)-\mu=0$ and $\mu=P(\lambda) \in \sigma^{*}(P(\mathbf{a}))$. So $\sigma^{*}(P(\mathbf{a})) \subset P\left(\sigma^{*}(\mathbf{a})\right)$ and we have shown that the one way finite polynomial spectral mapping property and the projection property imply the finite polynomial spectral mapping property.

Our first proposition, Theorem 2.3 in [17], says that spectral systems always extend from finite to infinite collections.

Proposition 1. If $\sigma^{*}$ is a spectral system for $\mathfrak{C}_{0}(\mathcal{A})$ then there exists a unique mapping, that we still denote by $\sigma^{*}$, which maps $\left(a_{i}\right)_{i \in I} \in \mathfrak{C}(\mathcal{A})$ onto a compact subset of $\mathbb{C}^{I}$ satisfying the projection property for infinite systems and whose restriction to $\mathfrak{C}_{0}(\mathcal{A})$ coincides with $\sigma^{*}$.

By (a) and (b) of Definition 1 and by Proposition 1 the spectrum of $\mathbf{a}:=\left(a_{i}\right)_{i \in I} \in \mathfrak{C}(\mathcal{A})$ is a non-empty compact subset of $\mathbb{C}^{I}$.

The projection property and the finite polynomial spectral mapping property can be combined to transfer polynomial identities between elements in $\mathbf{a}:=\left(a_{i}\right)_{i \in I}$ and $\lambda:=\left(\lambda_{i}\right)_{i \in I} \in \sigma^{*}(\mathbf{a})$. The method is very simple and useful (see [10] and Theorem 11.4.3 in [11]). We give the full details in one case in Lemma 1 and sufficient details in the other cases that we require.

Lemma 1. Let $\mathbf{a}=\left(a_{i}\right)_{i \in I} \in \mathfrak{C}(\mathcal{A})$ where $\mathcal{A}$ is a unital Banach algebra. Let $\sigma^{*}$ denote a spectral system and suppose $\lambda:=\left(\lambda_{i}\right)_{i \in I} \in \sigma^{*}(\mathbf{a})$. If $i, j, k \in I$ then the following are true:

(i) If $a_{i}+a_{j}=a_{k}$ then $\lambda_{i}+\lambda_{j}=\lambda_{k}$.

(ii) If $a_{i}=a_{j}$ then $\lambda_{i}=\lambda_{j}$.

(iii) If $a_{i} \cdot a_{j}=a_{k}$ then $\lambda_{i} \cdot \lambda_{j}=\lambda_{k}$.

(iv) If $\alpha \in \mathbb{C}$ and $\alpha a_{i}=a_{j}$ then $\alpha \lambda_{i}=\lambda_{j}$.

Proof. (i) Let $\pi\left(\left(a_{i}\right)_{i \in I}\right)=\left(a_{i}, a_{j}, a_{k}\right)$ and $P\left(z_{i}, z_{j}, z_{k}\right)=z_{i}+z_{j}-z_{k}$. By the projection property $\left(\lambda_{i}, \lambda_{j}, \lambda_{k}\right) \in \sigma^{*}(\mathbf{a})$ and by the finite polynomial spectral mapping property

$$
\begin{aligned}
P\left(\lambda_{i}, \lambda_{j}, \lambda_{k}\right) & =\lambda_{i}+\lambda_{j}-\lambda_{k} \\
& \in \sigma^{*}\left(P\left(\left\{a_{i}, a_{j}, a_{k}\right\}\right)=\sigma^{*}\left(\left\{a_{i}+a_{j}-a_{k}\right\}\right)=\sigma(\{0\})=0 .\right.
\end{aligned}
$$


Hence $P\left(\lambda_{i}, \lambda_{j}, \lambda_{k}\right)=\lambda_{i}+\lambda_{j}-\lambda_{k}=0$. For (ii) use $P\left(z_{i}, z_{j}\right)=z_{i}-z_{j}$, for (iii) use $P\left(z_{i}, z_{j}, z_{k}\right)=z_{i} \cdot z_{j}-z_{k}$, and for (iv) use $P\left(z_{i}, z_{j}\right)=\alpha z_{i}-z_{j}$ and the method used to prove (i). This completes the proof.

Our next result is Theorem 3.3 in [17]. We include a simple proof based on Lemma 1.

Proposition 2. Let $\sigma^{*}$ denote a spectral system. If $\mathcal{B}$ is a closed commutative unital subalgebra of the unital Banach algebra $\mathcal{A}$ then there exists $\mathcal{S}^{*}(\mathcal{B}) \subset \mathcal{S}(\mathcal{B})$ such that

$$
\sigma^{*}\left(\left(a_{i}\right)_{i \in I}\right)=\left\{\left(m\left(a_{i}\right)\right)_{i \in I}: m \in \mathcal{S}^{*}(\mathcal{B})\right\}
$$

for all $\left(a_{i}\right)_{i \in I} \subset \mathcal{B}$.

Proof. If $\mathbf{b}:=\mathcal{B}=\left(b_{j}\right)_{j \in J}$ for some indexing set $J$ and $\lambda:=\left(\lambda_{j}\right)_{j \in J} \in$ $\sigma^{*}(\mathbf{b})$, let $\widetilde{\lambda}: \mathcal{B} \rightarrow \mathbb{C}$ be defined by $\widetilde{\lambda}\left(b_{j}\right)=\lambda_{j}$. By (i), (iii), and (iv) above, $\tilde{\lambda} \in \mathcal{S}(\mathcal{B})$. Let $\mathcal{S}^{*}(\mathcal{B}):=\left\{\tilde{\lambda}: \lambda \in \sigma^{*}(\mathcal{B})\right\}$. Then $\sigma^{*}(\mathbf{b})=\left\{\left(m\left(b_{j}\right)\right)_{j \in J}: m \in\right.$ $\left.\mathcal{S}^{*}(\mathcal{B})\right\}$.

Let $\mathbf{a}=\left(a_{i}\right)_{i \in I}$ and suppose $a_{i} \in \mathcal{B}$ for all $i$ and let $\mathbf{c}=(\mathbf{a}, \mathbf{b})$. Then $\mathbf{c} \in \mathfrak{C}(\mathcal{A})$. By the projection property $\left(\lambda_{i}\right)_{i \in I} \in \sigma^{*}(\mathbf{a})$ if and only if there exists $\left(\beta_{j}\right)_{j \in J} \in \sigma^{*}(\mathcal{B})$ such that $\left(\left(\lambda_{i}\right)_{i \in I},\left(\beta_{j}\right)_{j \in J}\right) \in \sigma^{*}(\mathbf{c})$. By the above, there exists $m \in \mathcal{S}^{*}(\mathcal{B})$ such that $\beta_{j}=m\left(b_{j}\right)$ for all $j \in J$. If $i \in I$ then there exist $j \in J$ such that $a_{i}=b_{j}$. Hence $\lambda_{i}=\beta_{j}=m\left(a_{i}\right)=m\left(b_{j}\right)$ and $\left(\lambda_{i}\right)_{i \in I}=\left(m\left(a_{i}\right)\right)_{i \in I}$. Conversely suppose $m \in \mathcal{S}^{*}(\mathcal{B})$. Then $\left(m\left(b_{j}\right)\right)_{j \in J} \in$ $\sigma^{*}(\mathbf{b})$ and by the projection property there exists $\left(\lambda_{i}\right)_{i \in I} \in \sigma^{*}(\mathbf{a})$ such that $\left(\left(\lambda_{i}\right)_{i \in I},\left(m\left(b_{j}\right)_{j \in J}\right)\right) \in \sigma^{*}(\mathbf{c})$. As above, $\lambda_{i}=m\left(a_{i}\right)$ for all $i \in I$ and by the projection property $\left(m\left(a_{i}\right)\right)_{i \in I} \in \sigma^{*}(\mathbf{a})$. This completes the proof.

3. Tensor products. We now introduce the setting in which we will operate. To avoid the excessive use of parentheses we use interchangeably the following functional notation: $(f) x:=f(x)=:(f)(x)$.

Definition 2. Let $\gamma$ denote a uniform cross-norm, let $X$ denote a Banach space and let $\mathcal{A}$ denote a Banach algebra with identity e. If a $\in$ $\mathcal{A} \widehat{\otimes}_{\gamma} X$ we let $\mathcal{A}_{\mathbf{a}}$ denote the closed unital subalgebra of $\mathcal{A}$ generated by $\left(\left(\mathbf{e} \otimes x^{\prime}\right) \mathbf{a}\right)_{x^{\prime} \in X^{\prime}}$ and call $\mathcal{A}_{\mathbf{a}}$ the algebra generated by $\mathbf{a}$. If $\mathcal{A}_{\mathbf{a}}$ is commutative we say that a is commutative. For a commutative in $\mathcal{A} \widehat{\otimes}_{\gamma} X$ and $\sigma^{*}$ a spectral system let

$$
\sigma^{*}(\mathbf{a})=\sigma^{*}\left(\left(\left(\mathbf{e} \otimes x^{\prime}\right) \mathbf{a}\right)_{x^{\prime} \in X^{\prime}}\right) .
$$

If $\lambda \in \sigma^{*}(\mathbf{a})$ then $\lambda \in \mathbb{C}^{X^{\prime}}$ and there exists a unique mapping $\widetilde{\lambda}: X^{\prime} \rightarrow \mathbb{C}$ such that $\lambda_{x^{\prime}}=\widetilde{\lambda}\left(x^{\prime}\right)$ for all $x^{\prime} \in X^{\prime}$. By Lemma $1, \widetilde{\lambda}$ is a linear mapping on $X^{\prime}$. Since $\sigma^{*}\left(\left(\mathbf{e} \otimes x^{\prime}\right) \mathbf{a}\right) \subset \sigma\left(\left(\mathbf{e} \otimes x^{\prime}\right) \mathbf{a}\right)$ and $\left\|\left(\mathbf{e} \otimes x^{\prime}\right) \mathbf{a}\right\| \leq\left\|x^{\prime}\right\| \cdot\|\mathbf{a}\|$ we have $\left|\widetilde{\lambda}\left(x^{\prime}\right)\right| \leq\|\mathbf{a}\| \cdot\left\|x^{\prime}\right\|$ for all $x^{\prime} \in X^{\prime}$. Hence $\widetilde{\lambda} \in X^{\prime \prime}$ and $\|\widetilde{\lambda}\| \leq\|\mathbf{a}\|$. 
To refine this result we need the following lemma about arbitrary uniform cross-norms on Banach spaces.

Lemma 2. If $\gamma$ is a uniform cross-norm and $\mathbf{a} \in X \widehat{\otimes}_{\gamma} Y$ where $X$ and $Y$ are Banach spaces then the mappings

$$
\begin{array}{ll}
T_{\mathbf{a}}^{l}:\left(X^{\prime}, \sigma\left(X^{\prime}, X\right)\right) \rightarrow Y, & T_{\mathbf{a}}^{l}(\phi)=\left(\phi \otimes \mathbf{1}_{Y}\right) \mathbf{a}, \\
T_{\mathbf{a}}^{r}:\left(Y^{\prime}, \sigma\left(Y^{\prime}, Y\right)\right) \rightarrow X, & T_{\mathbf{a}}^{r}(\theta)=\left(\mathbf{1}_{X} \otimes \theta\right) \mathbf{a}
\end{array}
$$

are uniformly continuous on bounded sets.

Proof. It suffices to consider the mapping $T_{\mathbf{a}}^{l}$. Let $\mathbf{a}:=\sum_{i=1}^{n} x_{i} \otimes y_{i} \in$ $X \otimes Y$. If $\left(\phi_{\alpha}\right)_{\alpha}$ and $\phi_{\alpha} \stackrel{\omega^{*}}{\longrightarrow} \phi$ as $\alpha \rightarrow \infty$ then

$$
T_{\mathbf{a}}^{l}\left(\phi_{\alpha}\right)=\sum_{i=1}^{n} \phi_{\alpha}\left(x_{i}\right) y_{i} \rightarrow \sum_{i=1}^{n} \phi\left(x_{i}\right) y_{i}=T_{\mathbf{a}}^{l}(\phi)
$$

and $T_{\mathbf{a}}^{l}$ is continuous on $\left(X^{\prime}, \sigma\left(X^{\prime}, X\right)\right)$.

Now suppose $\mathbf{a} \in X \widehat{\otimes}_{\gamma} Y, \phi_{\alpha} \in X^{\prime},\left\|\phi_{\alpha}\right\| \leq M$ and $\phi_{\alpha} \stackrel{\omega^{*}}{\longrightarrow} \phi$ as $\alpha \rightarrow \infty$. Let $\varepsilon>0$ be arbitrary. Choose $\mathbf{b} \in X \otimes Y$ such that $\|\mathbf{a}-\mathbf{b}\| \leq \varepsilon$. Then

$$
\begin{aligned}
\left\|T_{\mathbf{a}}^{l}\left(\phi_{\alpha}\right)-T_{\mathbf{a}}^{l}(\phi)\right\| & =\left\|\left(\phi_{\alpha} \otimes \mathbf{1}_{Y}\right) \mathbf{a}-\left(\phi \otimes \mathbf{1}_{Y}\right) \mathbf{a}\right\|=\left\|\left(\left(\phi_{\alpha}-\phi\right) \otimes \mathbf{1}_{Y}\right) \mathbf{a}\right\| \\
& \leq\left\|\left(\left(\phi_{\alpha}-\phi\right) \otimes \mathbf{1}_{Y}\right) \mathbf{b}\right\|+\left\|\left(\left(\phi_{\alpha}-\phi\right) \otimes \mathbf{1}_{Y}\right)(\mathbf{a}-\mathbf{b})\right\| \\
& \leq\left\|\left(\left(\phi_{\alpha}-\phi\right) \otimes \mathbf{1}_{Y}\right) \mathbf{b}\right\|+2 M \varepsilon .
\end{aligned}
$$

Since $\mathbf{b} \in X \otimes Y,\left(\left(\phi_{\alpha}-\phi\right) \otimes \mathbf{1}_{Y}\right) \mathbf{b} \rightarrow 0$. Hence $T_{\mathbf{a}}^{l}\left(\phi_{\alpha}\right) \rightarrow T_{\mathbf{a}}^{l}(\phi)$ as $\alpha \rightarrow \infty$ and this completes the proof.

If $X$ is a Banach space we let $J_{X}$ denote the canonical mapping from $X$ into $X^{\prime \prime}$.

Proposition 3. If $\mathbf{a} \in \mathcal{A} \widehat{\otimes}_{\gamma} X$ is commutative then there exists a nonempty norm compact subset $A \subset\{x \in X:\|x\| \leq\|\mathbf{a}\|\}$ such that $J_{X}(A)=$ $\sigma^{*}(\mathbf{a})$.

Proof. Let $x^{\prime \prime} \in \sigma^{*}(\mathbf{a}) \subset X^{\prime \prime}$ and let $\left(\phi_{\alpha}\right)_{\alpha}$ denote a bounded net in $X^{\prime}$ which converges $w^{*}$ to $\phi$. Then

$$
x^{\prime \prime}\left(\phi_{\alpha}\right)-x^{\prime \prime}(\phi)=x^{\prime \prime}\left(\phi_{\alpha}-\phi\right) \in \sigma^{*}\left(\left(\mathbf{e} \otimes\left(\phi_{\alpha}-\phi\right)\right) \mathbf{a}\right)
$$

and

$$
\left|x^{\prime \prime}\left(\phi_{\alpha}\right)-x^{\prime \prime}(\phi)\right| \leq\left\|\left(\mathbf{e} \otimes\left(\phi_{\alpha}-\phi\right)\right) \mathbf{a}\right\|=\left\|T_{\mathbf{a}}^{r}\left(\phi_{\alpha}-\phi\right)\right\| \rightarrow 0
$$

as $\alpha \rightarrow \infty$. Hence $x^{\prime \prime}$ is $w^{*}$ continuous on bounded sets and thus belongs to $J_{X}(X)$.

From now on we identify $\sigma^{*}(\mathbf{a})$ with a subset of $X$. This means that $x \in X$ belongs to $\sigma^{*}(\mathbf{a})$ if and only if $x^{\prime}(x) \in \sigma^{*}\left(\left(\mathbf{e} \otimes x^{\prime}\right) \mathbf{a}\right)$ for all $x^{\prime} \in X^{\prime}$.

Having already noted that $\sigma^{*}(\mathbf{a}) \subset\{x \in X:\|x\| \leq\|\mathbf{a}\|\}$ it now suffices to show that $\sigma^{*}(\mathbf{a})$ is norm compact. Let $x_{0} \in \sigma^{*}(\mathbf{a})$ and let $\phi: \mathcal{A} \rightarrow \mathbb{C}$ 
be given by $\phi(a)=x^{\prime}\left(x_{0}\right)$ if $a=\left(\mathbf{e} \otimes x^{\prime}\right) \mathbf{a}$. If $x^{\prime}$ and $y^{\prime}$ belong to $X^{\prime}$ and $\left(\mathbf{e} \otimes x^{\prime}\right) \mathbf{a}=\left(\mathbf{e} \otimes y^{\prime}\right) \mathbf{a}$ then $\left(\mathbf{e} \otimes\left(x^{\prime}-y^{\prime}\right)\right) \mathbf{a}=0$. Since $x_{0} \in \sigma^{*}(\mathbf{a})$, $\left(x^{\prime}-y^{\prime}\right) x_{0} \in \sigma^{*}\left(\left(\mathbf{e} \otimes\left(x^{\prime}-y^{\prime}\right)\right) \mathbf{a}\right)=\sigma^{*}(\{0\})$. Hence $\left(x^{\prime}-y^{\prime}\right) x_{0}=0$ and $\phi$ is well defined.

If $a_{i}=\left(\mathbf{e} \otimes x_{i}^{\prime}\right) \mathbf{a}$ and $\alpha_{i} \in \mathbb{C}$ for $i=1, \ldots, n$ then

$$
\sum_{i=1}^{n} \alpha_{i} \cdot a_{i}=\sum_{i=1}^{n} \alpha_{i} \cdot\left(\mathbf{e} \otimes x_{i}^{\prime}\right) \mathbf{a}=\left(\mathbf{e} \otimes\left(\sum_{i=1}^{n} \alpha_{i} \cdot x_{i}^{\prime}\right)\right) \mathbf{a}
$$

and $\phi$ is defined on a subspace of $\mathcal{A}$. Since

$$
\phi\left(\sum_{i=1}^{n} \alpha_{i} \cdot a_{i}\right)=\left(\sum_{i=1}^{n} \alpha_{i} \cdot x_{i}^{\prime}\right) x_{0}=\sum_{i=1}^{n} \alpha_{i} \cdot \phi\left(a_{i}\right),
$$

$\phi$ is linear. If $a=\left(\mathbf{e} \otimes x^{\prime}\right) \mathbf{a}$ then $\phi(a) \subset \sigma^{*}\left(\left(\mathbf{e} \otimes x^{\prime}\right) \mathbf{a}\right) \subset \sigma(a) \subset\{\lambda \in \mathbb{C}$ : $|\lambda| \leq\|a\|\}$. Hence $|\phi(a)| \leq\|a\|$ and $\|\phi\| \leq 1$.

By the Hahn-Banach theorem $\phi$ can be extended to $\mathcal{A}$ as a continuous linear mapping with the same norm. We choose an extension and denote it also by $\phi$.

If $x^{\prime} \in X^{\prime}, x \in X, b \in \mathcal{A}$ and $\theta \in \mathcal{A}^{\prime}$ then

$$
\theta\left(\left(\mathbf{e} \otimes x^{\prime}\right)(b \otimes x)\right)=\theta(b) \cdot x^{\prime}(x)
$$

and

$$
x^{\prime}\left(\left(\theta \otimes \mathbf{1}_{X}\right)(b \otimes x)\right)=x^{\prime}(\theta(b) x)=\theta(b) \cdot x^{\prime}(x) .
$$

On taking finite sums and using continuity we obtain

$$
\theta\left[\left(\mathbf{e} \otimes x^{\prime}\right)(\mathbf{b})\right]=x^{\prime}\left(\left(\theta \otimes \mathbf{1}_{X}\right) \mathbf{b}\right)
$$

for all $\mathbf{b} \in \mathcal{A} \widehat{\otimes}_{\gamma} X$. Applying this identity to $\phi$ and $a=\left(\mathbf{e} \otimes x^{\prime}\right) \mathbf{a}$ we obtain $x^{\prime}\left(x_{0}\right)=\phi\left(\left(\mathbf{e} \otimes x^{\prime}\right) \mathbf{a}\right)=x^{\prime}\left(\left(\phi \otimes \mathbf{1}_{X}\right) \mathbf{a}\right)$ for all $x^{\prime} \in X^{\prime}$. By the Hahn-Banach theorem $x_{0}=\left(\phi \otimes \mathbf{1}_{X}\right) \mathbf{a}=T_{\mathbf{a}}^{l}(\phi)$. Hence $\sigma^{*}(\mathbf{a}) \subset T_{\mathbf{a}}^{l}\left(B_{\mathcal{A}^{\prime}}(1)\right)$. By Lemma 2, $\sigma^{*}(\mathbf{a})$ is a relatively norm compact subset of $X$. If $x_{n} \in \sigma^{*}(\mathbf{a}) \stackrel{\|\cdot\|}{\longrightarrow} x$ as $n \rightarrow \infty$ then $x_{n} \rightarrow x$ weakly and hence $x_{n} \rightarrow x$ in $\mathbb{C}^{X^{\prime}}$. By Definition 1 , $x \in \sigma^{*}(\mathbf{a})$. Hence $\sigma^{*}(\mathbf{a})$ is closed and this completes the proof.

If $0 \notin \sigma^{*}(\mathbf{a})$ we say that $\mathbf{a}$ is invertible. Since $\left(\mathbf{e} \otimes x^{\prime}\right) \mathbf{a}-x^{\prime}(x) \mathbf{e}=$ $\left(\mathbf{e} \otimes x^{\prime}\right)(\mathbf{a}-\mathbf{e} \otimes x)$ for all $x \in X$ and all $x^{\prime} \in X^{\prime}$ we see that $x \notin \sigma^{*}(\mathbf{a})$ if and only if $\mathbf{a}-\mathbf{e} \otimes x$ is invertible.

4. Algebraic polynomial extensions. If $X$ and $Y$ are vector spaces over $\mathbb{C}$ we let $\mathcal{P}_{\mathrm{a}}\left({ }^{n} X ; Y\right)$ denote the set of $n$-homogeneous polynomials from $X$ into $Y$. A polynomial is a finite sum of homogeneous polynomials and we denote by $\mathcal{P}_{\mathrm{a}}(X ; Y)$ the space of polynomials from $X$ to $Y$. If $P \in \mathcal{P}_{\mathrm{a}}\left({ }^{n} X ; Y\right)$ we let $P^{\vee}$ denote the unique symmetric $n$-linear form on $X^{n}$ associated with 
$P$ (see [4]). Universal properties of the tensor product imply (see $[1,7])$ the following result.

Proposition 4. If $X, Y, Z, W$ are vector spaces, $Q \in \mathcal{P}_{\mathrm{a}}\left({ }^{n} Z ; W\right)$ and $P \in \mathcal{P}_{\mathrm{a}}\left({ }^{n} X ; Y\right)$ then there exists a unique $Q \otimes P \in \mathcal{P}_{\mathrm{a}}\left({ }^{n} Z \otimes X ; W \otimes Y\right)$ such that

$(Q \otimes P)^{\vee}\left(z_{1} \otimes x_{1}, \ldots, z_{n} \otimes x_{n}\right)=Q^{\vee}\left(z_{1}, \ldots, z_{n}\right) \otimes P^{\vee}\left(x_{1}, \ldots, x_{n}\right)$ for $\left(z_{i}\right)_{i=1}^{n} \subset Z$ and $\left(x_{i}\right)_{i=1}^{n} \subset X$.

If $Z$ is an algebra $\mathcal{A}$ and $Q(a)=a^{n}$ for all $a \in \mathcal{A}$ then $Q \in \mathcal{P}_{\mathrm{a}}\left({ }^{n} \mathcal{A} ; \mathcal{A}\right)$ and we let $P_{\mathcal{A}}:=Q \otimes P$. Note that $P_{\mathcal{A}}(a \otimes x)=a^{n} \otimes P(x)$ for all $a \in \mathcal{A}$ and all $x \in X$. If we identify $X$ with the subspace $\mathbf{e} \otimes X$ of $\mathcal{A} \otimes X$ then we may regard $P_{\mathcal{A}}$ as an extension of $P$. If $\left\{a_{1}, \ldots, a_{n}\right\} \subset \mathcal{A}$ let

$$
s\left(a_{1}, \ldots, a_{n}\right)=\frac{1}{n !} \sum_{\sigma \in S_{n}} a_{\sigma(1)} \cdots a_{\sigma(n)}
$$

denote the symmetrization of $\left\{a_{1}, \ldots, a_{n}\right\}$ where $S_{n}$ is the set of all permutations of $\{1, \ldots, n\}$.

For parts (a) and (b) of the following proposition we refer to [7].

\section{PROPOSITION 5.}

(a) If $\mathbf{a}:=\sum_{i=1}^{t} a_{i} \otimes x_{i} \in \mathcal{A} \otimes X$ and $P \in \mathcal{P}_{\mathrm{a}}\left({ }^{n} X ; Y\right)$ then

$$
P_{\mathcal{A}}(\mathbf{a})=\sum_{k \in t^{n}} \mathbf{a}^{k} \otimes P^{\vee}\left(x^{k}\right)
$$

where $t^{n}=\left\{\left(i_{1}, \ldots, i_{n}\right): 1 \leq i_{j} \leq t\right\}, \mathbf{a}^{k}=a_{i_{1}} \cdots a_{i_{n}}$, and $x^{k}=$ $\left(x_{i_{1}}, \ldots, x_{i_{n}}\right)$ for $k=\left(i_{1}, \ldots, i_{n}\right)$.

(b) If $P \in \mathcal{P}_{\mathrm{a}}\left({ }^{n} X ; Y\right)$ then $P_{\mathcal{A}}$ is the unique polynomial in $\mathcal{P}_{\mathrm{a}}\left({ }^{n} \mathcal{A} \otimes X\right.$; $\mathcal{A} \otimes Y)$ such that

$$
\left[P_{\mathcal{A}}\right]^{\vee}\left(a_{1} \otimes x_{1}, \ldots, a_{n} \otimes x_{n}\right)=s\left(a_{1}, \ldots, a_{n}\right) \otimes P^{\vee}\left(x_{1}, \ldots, x_{n}\right)
$$

for any set $\left\{a_{i} \otimes x_{i}\right\}_{i=1}^{n} \subset \mathcal{A} \otimes X$.

(c) If $\phi \in X^{*}:=\mathcal{P}\left({ }^{1} X\right.$; $)$ then $\phi_{\mathcal{A}}=\mathbf{e} \otimes \phi$.

(d) If $\left\{\phi_{i}\right\}_{i=1}^{n} \subset X^{*}$ then

$$
\left(\phi_{1} \cdots \phi_{n}\right)_{\mathcal{A}}(\mathbf{a})=\frac{1}{n !} \sum_{\sigma \in S_{n}}\left(\phi_{\sigma(1)}\right)_{\mathcal{A}}(\mathbf{a}) \cdots\left(\phi_{\sigma(n)}\right)_{\mathcal{A}}(\mathbf{a}) .
$$

Proof. (c) If $a \in \mathcal{A}$ and $x \in X$ then $\phi_{\mathcal{A}}(a \otimes x)=a \otimes \phi(x)=(\mathbf{e} \otimes \phi)(a \otimes x)$. By linearity $\phi_{\mathcal{A}}=\mathbf{e} \otimes \phi$. 
(d) Clearly $\phi_{1} \cdots \phi_{n} \in \mathcal{P}_{\mathrm{a}}\left({ }^{n} X\right)$ and

$$
\begin{aligned}
\left(\phi_{1} \cdots \phi_{n}\right)^{\vee}\left(x_{1}, \ldots, x_{n}\right) & =\frac{1}{n !} \sum_{\sigma \in S_{n}} \phi_{1}\left(x_{\sigma(1)}\right) \cdots \phi_{n}\left(x_{\sigma(n)}\right) \\
& =\frac{1}{n !} \sum_{\sigma \in S_{n}} \phi_{\sigma(1)}\left(x_{1}\right) \cdots \phi_{\sigma(n)}\left(x_{n}\right) .
\end{aligned}
$$

If $\left\{\mathbf{a}_{i}\right\}_{i=1}^{n} \subset \mathcal{A} \otimes X$ let

$$
Q\left(\mathbf{a}_{1}, \ldots, \mathbf{a}_{n}\right)=\frac{1}{(n !)^{2}} \sum_{\sigma, \tau \in S_{n}}\left(\phi_{\sigma(1)}\right)_{\mathcal{A}}\left(\mathbf{a}_{\tau(1)}\right) \cdots\left(\phi_{\sigma(n)}\right)_{\mathcal{A}}\left(\mathbf{a}_{\tau(n)}\right) .
$$

The mapping $Q$ is easily seen to be symmetric $n$-linear. Some tedious but straightforward calculations, using parts (b), (c) and the formula for $\left(\phi_{1}, \ldots, \phi_{n}\right)^{\vee}$, show that

$$
Q\left(a_{1} \otimes x_{1}, \ldots, a_{n} \otimes x_{n}\right)=\left[\left(\phi_{1} \cdots \phi_{n}\right)_{\mathcal{A}}\right]^{\vee}\left(a_{1} \otimes x_{1}, \ldots, a_{n} \otimes x_{n}\right)
$$

when $\left\{a_{i} \otimes x_{i}\right\}_{i=1}^{n} \subset \mathcal{A} \otimes X$. Hence $Q=\left[\left(\phi_{1} \cdots \phi_{n}\right)_{\mathcal{A}}\right]^{\vee}$ and (3) follows easily. This completes the proof.

If $R: X \rightarrow Y$ is a linear mapping between vector spaces we let $R^{[n]}$ : $X^{n} \rightarrow Y^{n}$ denote the $n$-linear mapping defined by $R^{[n]}\left(x_{1}, \ldots, x_{n}\right)=$ $\left(R\left(x_{1}\right), \ldots, R\left(x_{n}\right)\right)$.

Lemma 3. If $P: X \rightarrow Y, R: Z \rightarrow W$ are linear mappings between vector spaces and $Q \in \mathcal{P}_{\mathrm{a}}\left({ }^{n} Y ; Z\right)$ then $(R \circ Q \circ P)_{\mathcal{A}}=R_{\mathcal{A}} \circ Q_{\mathcal{A}} \circ P_{\mathcal{A}}$.

Proof. If $\left(x_{i}\right)_{i=1}^{n} \subset X$ then, by the polarization formula [4],

$$
\begin{aligned}
(R \circ Q \circ P)^{\vee}\left(x_{1}, \ldots, x_{n}\right) & =\frac{1}{n ! 2^{n}} \sum_{\varepsilon_{i}= \pm 1} \varepsilon_{1} \cdots \varepsilon_{n}(R \circ Q \circ P)\left(\sum_{i=1}^{n} \varepsilon_{i} x_{i}\right) \\
& =\frac{1}{n ! 2^{n}} \sum_{\varepsilon_{i}= \pm 1} \varepsilon_{1} \cdots \varepsilon_{n}(R \circ Q)\left(\sum_{i=1}^{n} \varepsilon_{i} P\left(x_{i}\right)\right) \\
& =R\left(\frac{1}{n ! 2^{n}} \sum_{\varepsilon_{i}= \pm 1} \varepsilon_{1} \cdots \varepsilon_{n} Q\left(\sum_{i=1}^{n} \varepsilon_{i} P\left(x_{i}\right)\right)\right) \\
& =R\left(Q^{\vee}\left(P\left(x_{1}\right), \ldots, P\left(x_{n}\right)\right)\right) \\
& =R \circ Q^{\vee} \circ P^{[n]}\left(x_{1}, \ldots, x_{n}\right) .
\end{aligned}
$$

If $\left(x_{i}\right)_{i=1}^{n} \subset X$ and $\left(a_{i}\right)_{i=1}^{n} \subset \mathcal{A}$ then, using Proposition 5, 


$$
\begin{aligned}
{\left[(R \circ Q \circ P)_{\mathcal{A}}\right]^{\vee}\left(a_{1}\right.} & \otimes \\
& \left.=x_{1}, \ldots, a_{n} \otimes x_{n}\right) \\
& =s\left(a_{1}, \ldots, a_{n}\right) \otimes(R \circ Q \circ P)^{\vee}\left(x_{1}, \ldots, a_{n}\right) \otimes\left(R \circ x_{n}\right) \\
& =R_{\mathcal{A}}\left(s\left(a_{1} \cdots a_{n}\right) \otimes Q^{\vee n]}\left(P\left(x_{1}\right), \ldots, P\left(x_{1}, \ldots, x_{n}\right)\right)\right. \\
& =R_{\mathcal{A}}\left(\left(Q_{\mathcal{A}}\right)^{\vee}\left(a_{1} \otimes P\left(x_{1}\right), \ldots, a_{n} \otimes P\left(x_{n}\right)\right)\right) \\
& =\left(R_{\mathcal{A}} \circ\left(Q_{\mathcal{A}}\right)^{\vee} \circ\left(P_{\mathcal{A}}\right)^{[n]}\right)\left(a_{1} \otimes x_{1}, \ldots, a_{n} \otimes x_{n}\right) .
\end{aligned}
$$

Hence $\left[(R \circ Q \circ P)_{\mathcal{A}}\right]^{\vee}=R_{\mathcal{A}} \circ\left(Q_{\mathcal{A}}\right)^{\vee} \circ\left(P_{\mathcal{A}}\right)^{[n]}$ and

$$
\begin{aligned}
(R \circ Q \circ P)_{\mathcal{A}}(\mathbf{a}) & =\left[(R \circ Q \circ P)_{\mathcal{A}}\right]^{\vee}\left(\mathbf{a}^{n}\right)=R_{\mathcal{A}} \circ\left(Q_{\mathcal{A}}\right)^{\vee}\left(P_{\mathcal{A}}(\mathbf{a}), \ldots, P_{\mathcal{A}}(\mathbf{a})\right) \\
& =\left(R_{\mathcal{A}} \circ Q_{\mathcal{A}}\right)\left(P_{\mathcal{A}}(\mathbf{a})\right)=R_{\mathcal{A}} \circ Q_{\mathcal{A}} \circ P_{\mathcal{A}}(\mathbf{a}) .
\end{aligned}
$$

This completes the proof.

The above result is true for arbitrary $P$ and $Q$ if $\mathcal{A}$ is commutative [8] whereas the following example shows that it does not generally hold.

ExAmPle 1. If $H$ is a Hilbert space and $\mathcal{A}=\mathcal{B}(H), P\left(z_{1}, z_{2}\right)=z_{1} z_{2}$ for $\left(z_{1}, z_{2}\right) \in \mathbb{C}^{2}, Q(z)=z^{2}$ for $z \in \mathbb{C}$ and $e_{1}=(1,0)$ and $e_{2}=(0,1)$ denote the standard unit vector basis for $\mathbb{C}^{2}$, then

$$
\left(Q_{\mathcal{A}} \circ P_{\mathcal{A}}\right)\left(a \otimes e_{1}+b \otimes e_{2}\right)=\frac{1}{4}(a b+b a)^{2}
$$

while

$$
(Q \circ P)_{\mathcal{A}}\left(a \otimes e_{1}+b \otimes e_{2}\right)=\frac{1}{6}(a a b b+a b a b+a b b a+b a b a+b a a b+b b a a) .
$$

If $a$ denotes the unilateral shift and $b=a^{*}$ then

$$
(Q \circ P)_{\mathcal{A}}\left(a \otimes e_{1}+b \otimes e_{2}\right) \neq\left(Q_{\mathcal{A}} \circ P_{\mathcal{A}}\right)\left(a \otimes e_{1}+b \otimes e_{2}\right) .
$$

5. Continuous polynomial extensions. In this section we extend the algebraic results of the previous section to continuous polynomials on completed tensor products.

Let $\mathcal{P}\left({ }^{n} X ; Y\right)$ denote the space of all continuous $n$-homogeneous polynomials from the Banach space $X$ to the Banach space $Y$ endowed with the topology of uniform convergence over the unit ball of $X$ and let $\mathcal{P}(X ; Y)$ denote the space of all continuous polynomials.

If $\mathcal{A}$ is a unital Banach algebra, $\gamma$ is a uniform cross-norm, $X$ and $Y$ are Banach spaces and $P \in \mathcal{P}(X ; Y)$ satisfies

$$
\sup \left\{\left\|P_{\mathcal{A}}(\theta)\right\|: \theta \in \mathcal{A} \otimes X,\|\theta\|_{\gamma} \leq 1\right\}<\infty,
$$

then $P_{\mathcal{A}}$ extends to define a element in $\mathcal{P}\left({ }^{n}\left(\mathcal{A} \widehat{\otimes}_{\gamma} X\right) ; \mathcal{A} \widehat{\otimes}_{\gamma} Y\right)$. In this case we say that $P$ can be adapted to $\mathcal{A} \widehat{\otimes}_{\gamma} X$. 
Proposition 6. If $\mathcal{A}$ is a unital Banach algebra, $\gamma$ is a uniform crossnorm, $X$ is a Banach space and $\left\{\phi_{i}\right\}_{i=1}^{n} \subset X^{\prime}$, then $\left(\phi_{1} \cdots \phi_{n}\right)_{\mathcal{A}} \in$ $\left.\mathcal{P}{ }^{n}\left(\mathcal{A} \widehat{\otimes}_{\gamma} X\right) ; \mathcal{A} \widehat{\otimes}_{\gamma} Y\right)$ and

$$
\left(\phi_{1} \cdots \phi_{n}\right)_{\mathcal{A}}(\mathbf{a})=\frac{1}{n !} \sum_{\sigma \in S_{n}}\left(\phi_{\sigma(1)}\right)_{\mathcal{A}}(\mathbf{a}) \cdots\left(\phi_{\sigma(n)}\right)_{\mathcal{A}}(\mathbf{a}) .
$$

If $\mathbf{a}$ is commutative then

$$
\left(\phi_{1} \cdots \phi_{n}\right)_{\mathcal{A}}(\mathbf{a})=\left(\phi_{1}\right)_{\mathcal{A}}(\mathbf{a}) \cdots\left(\phi_{n}\right)_{\mathcal{A}}(\mathbf{a}) .
$$

Proof. For (4) it suffices to apply continuity and (3) in Proposition 5, and for (5) the definition of a commutative element and (4) complete the proof.

Definition 3 below was introduced in [8] (see also [5]) and shown to be necessary for the existence of a $\tau_{0}$-continuous functional calculus for all $\mathbf{a} \in$ $\mathcal{A} \widehat{\otimes}_{\gamma} X$ when $\mathcal{A}$ is commutative, $X$ is some infinite-dimensional Banach space and $\gamma$ is a uniform cross-norm.

Definition 3. Let $\mathcal{A}$ denote a unital Banach algebra and let $\gamma$ denote a uniform cross-norm. A Banach space $X$ has the $(\mathcal{A}, \gamma)$-extension property if every $P \in \mathcal{P}(X)$ can be adapted to $\mathcal{A} \widehat{\otimes}_{\gamma} X$ and there exists $c \geq 1$ such that for all $n$ and all $P \in \mathcal{P}\left({ }^{n} X\right)$,

$$
\left\|P_{\mathcal{A}}\right\| \leq c^{n}\|P\| \text {. }
$$

In this article we are more involved with a commutative subalgebra of a non-commutative algebra and give a more general definition, Definition 4 , which focuses on particular elements in $\mathcal{A} \widehat{\otimes}_{\gamma} X$ rather than on the whole space. We use the following notation from now on: if $\mathcal{A}$ is a unital Banach algebra, $\gamma$ is a uniform cross-norm, $X$ is a Banach space and a belongs to $\mathcal{A} \widehat{\otimes}_{\gamma} X$, then $\|\cdot\|_{\gamma^{\prime}}$ is the norm on $\mathcal{A}_{\mathbf{a}} \otimes X$ induced by the norm $\|\cdot\|_{\gamma}$ on $\mathcal{A} \widehat{\otimes}_{\gamma} X$. In general, the canonical mapping from $\mathcal{A}_{\mathbf{a}} \widehat{\otimes}_{\gamma} X$ into $\mathcal{A} \widehat{\otimes}_{\gamma} X$ is not an isomorphism onto its image, it will be injective when $X$ has the approximation property and it will always be continuous, since $\gamma$ is a uniform cross-norm, with norm less than or equal to 1 . This means that $\|\cdot\|_{\gamma^{\prime}} \leq\|\cdot\|_{\gamma}$ on $\mathcal{A}_{\mathbf{a}} \otimes X$ for any $\gamma$ and any $\mathbf{a} \in \mathcal{A} \widehat{\otimes}_{\gamma} X$. Since there is no possibility of confusion we let $\mathcal{A}_{\mathbf{a}} \widehat{\otimes}_{\gamma^{\prime}} X$ denote the closure of $\mathcal{A}_{\mathbf{a}} \otimes X$ in $\mathcal{A} \widehat{\otimes}_{\gamma} X$. If $\varepsilon$ is the injective tensor norm then $\mathcal{A}_{\mathbf{a}} \widehat{\otimes}_{\varepsilon^{\prime}} X=\mathcal{A}_{\mathbf{a}} \widehat{\otimes}_{\varepsilon} X$ for any $\mathbf{a} \in \mathcal{A} \widehat{\otimes}_{\varepsilon} X$.

Definition 4. If $\mathcal{A}$ is a unital Banach algebra, $\gamma$ is a uniform cross-norm, $X$ is a Banach space and a belongs to $\mathcal{A} \widehat{\otimes}_{\gamma} X$ then $X$ is $(\mathbf{a}, \gamma)$ adaptable if there exists $c \in \mathbb{R}$ such that for all $n$, for every Banach space $Y$ and all $P \in \mathcal{P}\left({ }^{n} X ; Y\right)$ we have

$$
\sup \left\{\left\|P_{\mathcal{A}}(\theta)\right\|: \theta \in \mathcal{A}_{\mathbf{a}} \otimes X,\|\theta\|_{\gamma} \leq 1\right\} \leq c^{n}\|P\| .
$$


When (6) holds, the restriction of $P_{\mathcal{A}}$ to $\mathcal{A}_{\mathbf{a}} \otimes X$ extends to define a continuous $\mathcal{A} \widehat{\otimes}_{\gamma} Y$-valued $n$-homogeneous polynomial, that we still denote by $P_{\mathcal{A}}$, on $\mathcal{A}_{\mathbf{a}} \widehat{\otimes}_{\gamma^{\prime}} X$.

Results in $[7,8]$ imply that any Banach space $X$ is $(\mathbf{a}, \pi)$ adaptable for all $\mathbf{a} \in \mathcal{A} \widehat{\otimes}_{\pi} X, \mathcal{A}$ arbitrary and $\pi$ the projective tensor product, and $(\mathbf{a}, \varepsilon)$ adaptable for all $\mathbf{a} \in \mathcal{U} \widehat{\otimes}_{\varepsilon} X$ where $\mathcal{U}$ is a uniform Banach algebra with identity and $\varepsilon$ is the injective tensor product.

ExAmple 2. If $\mathcal{A}=\mathcal{B}(\mathcal{H})$ and $\mathcal{C}$ is a unital $\mathcal{C}^{*}$-subalgebra of $\mathcal{B}(\mathcal{H})$ generated by a commuting family of normal operators, then $\mathcal{C}$ is isometrically isomorphic to a uniform algebra and $\mathcal{C} \widehat{\otimes}_{\varepsilon} X$ is isometrically isomorphic to a subspace of $\mathcal{B}(\mathcal{H}) \widehat{\otimes}_{\varepsilon} X$ for any Banach space $X$. By Example 6 in [7], $X$ is $(\mathbf{a}, \varepsilon)$ adaptable for any $\mathbf{a} \in \mathcal{C}$.

We now restrict ourselves to a commutative element $\mathbf{a} \in \mathcal{A} \widehat{\otimes}_{\gamma} X$ and to Banach spaces with the bounded approximation property in order to use the collection $\left(\mathbf{e} \otimes x^{\prime}\right)_{x^{\prime} \in X^{\prime}}$ to obtain properties of a.

Proposition 7. If $\gamma$ is a uniform cross-norm, $X$ and $Y$ are Banach spaces, $T: X \rightarrow Y$ is a continuous linear operator, $\mathcal{A}$ is a Banach algebra with identity $\mathbf{e}$ and $\mathbf{a} \in \mathcal{A} \widehat{\otimes}_{\gamma} X$ is commutative then $(\mathbf{e} \otimes T) \mathbf{a}$ is commutative and $\mathcal{A}_{(\mathbf{e} \otimes T) \mathbf{a}} \subset \mathcal{A}_{\mathbf{a}}$. If $T$ is a finite rank operator from $X$ into $X$ then $(\mathbf{e} \otimes T) \mathbf{a} \in \mathcal{A}_{\mathbf{a}} \otimes X$.

Proof. If $y^{\prime} \in Y^{\prime}$ then

$$
\left(y^{\prime}\right)_{\mathcal{A}}((\mathbf{e} \otimes T) \mathbf{a})=\left(\mathbf{e} \otimes y^{\prime}\right)(\mathbf{e} \otimes T) \mathbf{a}=\left(\mathbf{e} \otimes\left(y^{\prime} \circ T\right)\right) \mathbf{a}=\left(y^{\prime} \circ T\right)_{\mathcal{A}} \mathbf{a} .
$$

Since $y^{\prime} \circ T \in X^{\prime}$ and $\mathbf{a}$ is commutative this implies that $\left(\mathbf{e} \otimes y^{\prime}\right)(\mathbf{e} \otimes T) \mathbf{a} \in \mathcal{A}_{\mathbf{a}}$ and hence $(\mathbf{e} \otimes T) \mathbf{a}$ is commutative.

If $T(x)=\sum_{i=1}^{n} x_{i}^{\prime}(x) x_{i}$ for all $x \in X$ where $\left(x_{i}^{\prime}\right)_{i=1}^{n} \subset X^{\prime}$ and $\left(x_{i}\right)_{i=1}^{n} \subset X$ then by first considering $a \otimes x$, then taking finite sums and finally using continuity, we see that

$$
(\mathbf{e} \otimes T) \mathbf{a}=\sum_{i=1}^{n}\left(\left(\mathbf{e} \otimes x_{i}^{\prime}\right) \mathbf{a}\right) \otimes x_{i} \in \mathcal{A}_{\mathbf{a}} \otimes X .
$$

This completes the proof.

Definition 5. A Banach space $X$ has the bounded approximation property if there exists a bounded set $\left(T_{\alpha}\right)_{\alpha \in \Gamma}$ of finite rank operators in $\mathcal{B}(X)$ which converges to the identity mapping on $X$ uniformly on compact subsets of $X$. If each $T_{\alpha}$ is a projection we say that $X$ has the bounded projection property.

The bounded projection property is also known as the $\pi$-property. A separable Banach space is a complemented subspace of a Banach space 
with the bounded approximation property if and only if it is a complemented subspace of a Banach space with the bounded projection property.

Proposition 8. If $X$ is a Banach space with the bounded approximation property, $\gamma$ is a uniform cross-norm, $\mathcal{A}$ is a Banach algebra with identity $\mathbf{e}$ and $\mathbf{a} \in \mathcal{A} \widehat{\otimes}_{\gamma} X$, then $\left(\mathbf{e} \otimes T_{\alpha}\right) \mathbf{a} \rightarrow \mathbf{a}$ as $\alpha \rightarrow \infty$. If $\mathbf{a}$ is commutative then $\mathbf{a} \in \mathcal{A}_{\mathbf{a}} \widehat{\otimes}_{\gamma^{\prime}} X$.

Proof. Suppose $\left\|T_{\alpha}\right\| \leq M$ for all $\alpha$. Let $\varepsilon>0$ be arbitrary. Choose $\mathbf{b}:=\sum_{i=1}^{n} b_{i} \otimes x_{i} \in \mathcal{A} \otimes X$ such that $\|\mathbf{a}-\mathbf{b}\| \leq \varepsilon$. Then

$$
\begin{aligned}
\left\|\left(\mathbf{e} \otimes\left(T_{\alpha}-I_{X}\right)\right) \mathbf{b}\right\| & =\left\|\sum_{i=1}^{n} b_{i} \otimes\left(T_{\alpha}-I_{X}\right) x_{i}\right\| \\
& \leq \sum_{i=1}^{n}\left\|b_{i}\right\| \cdot\left\|\left(T_{\alpha}-I_{X}\right)\left(x_{i}\right)\right\| \rightarrow 0
\end{aligned}
$$

as $\alpha \rightarrow \infty$. Hence

$$
\begin{aligned}
\left\|\left(\mathbf{e} \otimes T_{\alpha}\right) \mathbf{a}-\mathbf{a}\right\| & =\left\|\left(\mathbf{e} \otimes\left(T_{\alpha}-I_{X}\right)\right) \mathbf{a}\right\| \\
& \leq\left\|\left(\mathbf{e} \otimes\left(T_{\alpha}-I_{X}\right)\right) \mathbf{b}\right\|+\left\|\left(\mathbf{e} \otimes\left(T_{\alpha}-I_{X}\right)\right)(\mathbf{a}-\mathbf{b})\right\| \\
& \leq\left\|\left(\mathbf{e} \otimes\left(T_{\alpha}-I_{X}\right)\right) \mathbf{b}\right\|+(M+1) \varepsilon
\end{aligned}
$$

and $\left(\mathbf{e} \otimes T_{\alpha}\right) \mathbf{a} \rightarrow \mathbf{a}$ as $\alpha \rightarrow \infty$.

If $\mathbf{a}$ is commutative, Proposition 7 implies that $\left(\mathbf{e} \otimes T_{\alpha}\right) \mathbf{a} \in \mathcal{A}_{\mathbf{a}} \otimes X$ for all $\alpha$ and, by the first part of the proposition, $\mathbf{a} \in \mathcal{A}_{\mathbf{a}} \widehat{\otimes}_{\gamma^{\prime}} X$. This completes the proof.

Proposition 9. If $X$ and $Y$ are Banach spaces and $X$ has the bounded approximation property, $\gamma$ is a uniform cross-norm, $\mathcal{A}$ is a Banach algebra with identity $\mathbf{e}, \mathbf{a}$ is a commutative element in $\mathcal{A} \widehat{\otimes}_{\gamma} X, X$ is $(\mathbf{a}, \gamma)$ adaptable and $P \in \mathcal{P}(X ; Y)$, then $P_{\mathcal{A}}(\mathbf{a})$ is well defined, $\mathcal{A}_{P_{\mathcal{A}}(\mathbf{a})} \subset \mathcal{A}_{\mathbf{a}}$ and $P_{\mathcal{A}}(\mathbf{a})$ is commutative.

Proof. By linearity we may suppose that $P$ is $n$-homogeneous. By Proposition $8, \mathbf{a} \in \mathcal{A}_{\mathbf{a}} \widehat{\otimes}_{\gamma^{\prime}} X$ and since $X$ is $(\mathbf{a}, \gamma)$ adaptable, $P_{\mathcal{A}} \in \mathcal{P}\left({ }^{n}\left(\mathcal{A}_{\mathbf{a}} \widehat{\otimes}_{\gamma^{\prime}} X\right)\right.$; $\left.\mathcal{A} \widehat{\otimes}_{\gamma} Y\right)$ and $P_{\mathcal{A}}(\mathbf{a})$ is well defined. To complete the proof we must show that $\left(y^{\prime}\right)_{\mathcal{A}}\left(P_{\mathcal{A}}(\mathbf{a})\right) \in \mathcal{A}_{\mathbf{a}}$ for all $y^{\prime} \in Y^{\prime}$. Since $\mathcal{A}_{\mathbf{a}}$ is a closed subspace of the Banach space $\mathcal{A}$ it suffices, by Lemma 3 and Proposition 8, to show that

$$
\left(y^{\prime}\right)_{\mathcal{A}}\left(P_{\mathcal{A}}((\mathbf{e} \otimes T) \mathbf{a})\right)=\left(y^{\prime}\right)_{\mathcal{A}}\left((P \circ T)_{\mathcal{A}} \mathbf{a}\right)=\left(y^{\prime} \circ P \circ T\right)_{\mathcal{A}}(\mathbf{a})
$$

lies in $\mathcal{A}_{\mathbf{a}}$ for any continuous finite rank operator $T$ from $X$ into $X$. If $T(x)=\sum_{j=1}^{l} \theta_{j}(x) x_{j}$ where $x_{j} \in X$ and $\theta_{j} \in X^{\prime}$ for all $j$ then

$$
\left(y^{\prime} \circ P \circ T\right)(x)=\sum_{m=\left\{j_{1}, \ldots, j_{n}\right\}, 1 \leq j_{i} \leq l} \theta^{m}(x) \cdot y^{\prime}\left(P^{\vee}\left(x_{m}\right)\right)
$$


where $\theta^{m}=\theta_{j_{1}} \cdots \theta_{j_{n}}$ and $x_{m}=\left(x_{j_{1}}, \ldots, x_{j_{n}}\right)$. By Proposition 6 ,

$$
\left(y^{\prime} \circ P \circ T\right)_{\mathcal{A}}(\mathbf{a})=\sum_{m=\left\{j_{1}, \ldots, j_{n}\right\}, 1 \leq j_{i} \leq l} y^{\prime}\left(P^{\vee}\left(x_{m}\right)\right) \cdot \theta_{\mathcal{A}}^{m}(\mathbf{a})
$$

where $\theta_{\mathcal{A}}^{m}(\mathbf{a})=\left(\theta_{j_{1}}\right)_{\mathcal{A}}(\mathbf{a}) \cdots\left(\theta_{j_{n}}\right)_{\mathcal{A}}(\mathbf{a})$. Since each $\left(\theta_{j_{i}}\right)_{\mathcal{A}}(\mathbf{a})$ belongs to $\mathcal{A}_{\mathbf{a}}$, $\left(y^{\prime} \circ P \circ T\right)_{\mathcal{A}}(\mathbf{a}) \in \mathcal{A}_{\mathbf{a}}$. This completes the proof.

Proposition 10. If $X$ is a Banach space with the bounded approximation property, $\gamma$ is a uniform cross-norm, $\mathcal{A}$ is a Banach algebra with identity $\mathbf{e}, \mathbf{a} \in \mathcal{A} \widehat{\otimes}_{\gamma} X$ is commutative and $X$ is $(\mathbf{a}, \gamma)$ adaptable, then the mapping

$$
P \in \mathcal{P}(X) \mapsto P_{\mathcal{A}}(\mathbf{a})
$$

is an algebra homomorphism.

Proof. By linearity it suffices to show $(P \cdot Q)_{\mathcal{A}}(\mathbf{a})=P_{\mathcal{A}}(\mathbf{a}) \cdot Q_{\mathcal{A}}(\mathbf{a})$ for all $P \in \mathcal{P}\left({ }^{n} X\right)$ and all $Q \in \mathcal{P}\left({ }^{m} X\right)$. Let $\left(T_{\alpha}\right)_{\alpha}$ denote the set of finite rank operators on $X$ associated with the bounded approximation property. By Proposition 8,

$$
\begin{aligned}
(P \cdot Q)_{\mathcal{A}}(\mathbf{a}) & =\lim _{\alpha \rightarrow \infty}(P \cdot Q)_{\mathcal{A}}\left(\left(\mathbf{e} \otimes T_{\alpha}\right) \mathbf{a}\right)=\lim _{\alpha \rightarrow \infty}(P \cdot Q)_{\mathcal{A}}\left(\left(T_{\alpha}\right)_{\mathcal{A}} \mathbf{a}\right) \\
& =\lim _{\alpha \rightarrow \infty}\left((P \cdot Q) \circ T_{\alpha}\right)_{\mathcal{A}}(\mathbf{a})=\lim _{\alpha \rightarrow \infty}\left(\left(P \circ T_{\alpha}\right) \cdot\left(Q \circ T_{\alpha}\right)\right)_{\mathcal{A}}(\mathbf{a}) .
\end{aligned}
$$

Now $P \circ T_{\alpha}=\sum_{i} \theta_{i}^{n}$ and $Q \circ T_{\alpha}=\sum_{j} \phi_{j}^{m}$ where the sums are finite, $\theta_{i}^{n}=\theta_{i_{1}} \cdots \theta_{i_{n}}$ and $\phi_{j}^{m}=\phi_{j_{1}} \cdots \theta_{j_{m}}, \theta_{i} \in X^{\prime}$ and $\phi_{j} \in X^{\prime}$ for all $i$ and $j$. Hence

$$
\left(P \circ T_{\alpha}\right) \cdot\left(Q \circ T_{\alpha}\right)=\sum_{i, j} \theta_{i}^{n} \cdot \phi_{j}^{m} .
$$

Proposition 6 implies, since $\mathbf{a}$ is commutative,

$$
\begin{aligned}
\left(\left(P \circ T_{\alpha}\right) \cdot\left(Q \circ T_{\alpha}\right)\right)_{\mathcal{A}}(\mathbf{a}) & =\sum_{i, j}\left(\theta_{i}^{n}\right)_{\mathcal{A}}(\mathbf{a}) \cdot\left(\phi_{j}^{m}\right)_{\mathcal{A}}(\mathbf{a}) \\
& =\left(P \circ T_{\alpha}\right)_{\mathcal{A}}(\mathbf{a}) \cdot\left(Q \circ T_{\alpha}\right)_{\mathcal{A}}(\mathbf{a})
\end{aligned}
$$

for all $\alpha$. On letting $\alpha \rightarrow \infty$ we obtain, by Proposition $8,(P \cdot Q)_{\mathcal{A}}(\mathbf{a})=$ $P_{\mathcal{A}}(\mathbf{a}) \cdot Q_{\mathcal{A}}(\mathbf{a})$. This completes the proof.

6. Polynomial functional calculus. If $\sigma^{*}$ is a spectral system and $\mathbf{a} \in \mathcal{A} \widehat{\otimes}_{\gamma} X$ is commutative then Proposition 2 together with the identity $a^{\prime}\left(\left(\mathbf{e} \otimes x^{\prime}\right) \mathbf{b}\right)=x^{\prime}\left(\left(a^{\prime} \otimes \mathbf{1}_{X}\right) \mathbf{b}\right)$ for all $x^{\prime} \in X^{\prime}, a^{\prime} \in \mathcal{A}^{\prime}$ and $\mathbf{b} \in \mathcal{A} \widehat{\otimes}_{\gamma} X$ shows that there exists a subset $\mathcal{S}^{*}(\mathbf{a})$ of $\mathcal{S}\left(\mathcal{A}_{\mathbf{a}}\right)$ such that

$$
\sigma^{*}(\mathbf{b})=\left\{\left(m\left(\left(\mathbf{e} \otimes x^{\prime}\right) \mathbf{b}\right)\right)_{x^{\prime} \in X^{\prime}}: m \in \mathcal{S}^{*}(\mathbf{a})\right\}=\left\{\left(m \otimes \mathbf{1}_{X}\right) \mathbf{b}: m \in \mathcal{S}^{*}(\mathbf{a})\right\}
$$

whenever $\mathbf{b} \in \mathcal{A} \widehat{\otimes}_{\gamma} X$ satisfies $\left(\mathbf{e} \otimes x^{\prime}\right) \mathbf{b} \in \mathcal{A}_{\mathbf{a}}$ for all $x^{\prime} \in X^{\prime}$.

Proposition 11. Let $\mathcal{A}$ denote a Banach algebra with identity $\mathbf{e}, X$ a Banach space with the bounded approximation property, $\gamma$ a uniform cross- 
norm and let $\sigma^{*}$ denote a spectral system. If $\mathbf{a} \in \mathcal{A} \widehat{\otimes}_{\gamma} X$ is commutative and $X$ is $(\mathbf{a}, \gamma)$ adaptable then

$$
\sigma^{*}\left(P_{\mathcal{A}}(\mathbf{a})\right)=P\left(\sigma^{*}(\mathbf{a})\right)
$$

for all $P \in \mathcal{P}(X)$.

Proof. By the above we must show

$$
m\left(P_{\mathcal{A}}(\mathbf{a})\right)=P\left(\left(m \otimes \mathbf{1}_{X}\right) \mathbf{a}\right)
$$

for all $m \in \mathcal{S}^{*}(\mathbf{a})$. Let $\left(T_{\alpha}\right)_{\alpha}$ denote a bounded net of finite rank operators which converges to the identity uniformly on compact subsets of $X$. By Proposition 7, $\left(T_{\alpha}\right)_{\mathcal{A}}(\mathbf{a})=\left(\mathbf{e} \otimes T_{\alpha}\right)(\mathbf{a}) \in \mathcal{A}_{\mathbf{a}} \otimes X \subset \operatorname{Domain}\left(P_{\mathcal{A}}\right)$ and, by Proposition 8, (e $\left.\otimes T_{\alpha}\right) \mathbf{a} \rightarrow \mathbf{a}$ as $\alpha \rightarrow \infty$. Hence it suffices to prove (9) with a replaced by $T_{\mathcal{A}}(\mathbf{a})$ where $T: X \rightarrow X$ is a finite rank operator and by linearity we may also suppose that $P \in \mathcal{P}\left({ }^{n} X\right)$.

If $T(x)=\sum_{j=1}^{l} \theta_{j}(x) x_{j}$ where $x_{j} \in X$ and $\theta_{j} \in X^{\prime}$ for all $j$ then

$$
(P \circ T)(x)=\sum_{k=\left\{j_{1}, \ldots, j_{n}\right\}, 1 \leq j_{i} \leq l} \theta^{k}(x) P^{\vee}\left(x_{k}\right)
$$

where $\theta^{k}=\theta_{j_{1}} \cdots \theta_{j_{n}}$ and $x_{k}=\left(x_{j_{1}}, \ldots, x_{j_{n}}\right)$. By Proposition 6 ,

$$
P_{\mathcal{A}}\left(T_{\mathcal{A}}(\mathbf{a})\right)=(P \circ T)_{\mathcal{A}}(\mathbf{a})=\sum_{k=\left\{j_{1}, \ldots, j_{n}\right\}, 1 \leq j_{i} \leq l} P^{\vee}\left(x_{k}\right) \cdot \theta_{\mathcal{A}}^{k}(\mathbf{a})
$$

where $\theta_{\mathcal{A}}^{k}(\mathbf{a})=\left(\theta_{j_{1}}\right)_{\mathcal{A}}(\mathbf{a}) \cdots\left(\theta_{j_{n}}\right)_{\mathcal{A}}(\mathbf{a})$. Since $m$ and $\left(\theta_{j_{i}}\right)_{\mathcal{A}}$ are continuous linear mappings,

$$
m\left(\left(\theta_{j_{i}}\right)_{\mathcal{A}}(\mathbf{a})\right)=m\left(\left(\mathbf{e} \otimes \theta_{j_{i}}\right) \mathbf{a}\right)=\theta_{j_{i}}\left(\left(m \otimes \mathbf{1}_{X}\right) \mathbf{a}\right),
$$

and as $\left(\theta_{j_{i}}\right)_{\mathcal{A}}(\mathbf{a})$ belongs to $\mathcal{A}_{\mathbf{a}}$ for all $j_{i}$ and $m \in \mathcal{S}\left(\mathcal{A}_{\mathbf{a}}\right)$,

$$
\begin{aligned}
m\left(P_{\mathcal{A}}\left(T_{\mathcal{A}}(\mathbf{a})\right)\right) & =\sum_{k=\left\{j_{1}, \ldots, j_{n}\right\}, 1 \leq j_{i} \leq l} P^{\vee}\left(x_{k}\right) \cdot \theta^{k}\left(\left(m \otimes \mathbf{1}_{X}\right) \mathbf{a}\right) \\
& =P \circ T\left(\left(m \otimes \mathbf{1}_{X}\right) \mathbf{a}\right)=P\left(\left(m \otimes \mathbf{1}_{X}\right)(\mathbf{e} \otimes T) \mathbf{a}\right) \\
& =P\left(\left(m \otimes \mathbf{1}_{X}\right)\left(T_{\mathcal{A}}(\mathbf{a})\right)\right) .
\end{aligned}
$$

This completes the proof.

7. Holomorphic functional calculus. The results and proofs in this section are, in some cases, modified versions of those given in [8], to which we refer for details.

If $U$ is an open subset of a Banach space $X$ we let $\mathcal{H}(U)$ denote the space of holomorphic functions on $U$ and let $\tau_{0}$ denote the compact open topology on $\mathcal{H}(U)$. Let $\mathcal{H}_{\mathrm{b}}(U)$ denote the subspace of $\mathcal{H}(U)$ consisting of those functions which are bounded on the bounded subsets of $U$ which lie strictly inside $U$. Endowed with the topology, $\tau_{\mathrm{b}}$, of uniform convergence on 
these sets the space $\mathcal{H}_{\mathrm{b}}(U)$ is a Fréchet space (we refer to $[4,8]$ for further details). When $X$ is finite-dimensional, $\mathcal{H}_{\mathrm{b}}(U)=\mathcal{H}(U)$. We let $B_{r}=\{x \in$ $X:\|x\|<r\}$.

Lemma 4. Let $\mathcal{A}$ denote a Banach algebra with identity e, $X$ a Banach space with the bounded approximation property, $\gamma$ a uniform cross-norm and $\sigma^{*}$ a spectral system. If $\mathbf{a} \in \mathcal{A} \widehat{\otimes}_{\gamma} X$ is commutative, $X$ is $(\mathbf{a}, \gamma)$ adaptable, $c$ is a constant for which (6) holds and $r>c\|\mathbf{a}\|$, let $\theta_{\mathbf{a}}: \mathcal{H}_{\mathrm{b}}\left(B_{r}\right) \rightarrow \mathcal{A}_{\mathbf{a}}$,

$$
\theta_{\mathbf{a}}(f):=f_{\mathcal{A}}(\mathbf{a}):=\sum_{n=0}^{\infty}\left(P_{n}\right)_{\mathcal{A}}(\mathbf{a})
$$

where $\sum_{n=0}^{\infty} P_{n}$ is the Taylor series expansion of $f \in \mathcal{H}_{\mathrm{b}}\left(B_{r}\right)$. Then $\theta_{\mathrm{a}}$ is the unique $\tau_{\mathrm{b}}$ continuous algebra homomorphism from $\mathcal{H}_{\mathrm{b}}\left(B_{r}\right)$ into $\mathcal{A}$ which is $\tau_{0}$ continuous on the bounded subsets of $\mathcal{H}_{\mathrm{b}}\left(B_{r}\right)$ with the following property:

$$
\theta_{\mathbf{a}}\left(x^{\prime}\right)=x_{\mathcal{A}}^{\prime}(\mathbf{a})
$$

for all $x^{\prime} \in X^{\prime}$. Moreover,

$$
m\left(\theta_{\mathbf{a}}(f)\right)=f\left(\left(m \otimes \mathbf{1}_{X}\right) \mathbf{a}\right)
$$

for all $f \in \mathcal{H}\left(\sigma^{*}(\mathbf{a})\right)$ and all $m \in \mathcal{S}^{*}\left(\mathcal{A}_{\mathbf{a}}\right)$ and

$$
\sigma^{*}\left(\theta_{\mathbf{a}}(f)\right)=f\left(\sigma^{*}(\mathbf{a})\right)
$$

for all $f \in \mathcal{H}\left(\sigma^{*}(\mathbf{a})\right)$.

Proof. Existence, continuity and uniqueness follow as in Lemma 20 in [8], and Proposition 10 shows that $\theta_{\mathbf{a}}$ is an algebra homomorphism. Since $r>$ $c\|\mathbf{a}\|$ the series $\sum_{n=0}^{\infty}\left(P_{n}\right)_{\mathcal{A}}(\mathbf{a})$ converges and, by Proposition $9, f_{\mathcal{A}}(\mathbf{a}) \in \mathcal{A}_{\mathbf{a}}$ for all $x^{\prime} \in X^{\prime}$. By Propositions 2 and 9,

$$
\sigma^{*}\left(f_{\mathcal{A}}(\mathbf{a})\right)=\left\{\left(m \otimes \mathbf{1}_{X}\right) f_{\mathcal{A}}(\mathbf{a}): m \in \mathcal{S}^{*}(\mathbf{a})\right\} .
$$

If $m \in \mathcal{S}^{*}(\mathbf{a}),(9)$ and Proposition 11 imply

$$
m\left(f_{\mathcal{A}}(\mathbf{a})\right)=\sum_{n=0}^{\infty} m\left(\left(P_{n}\right)_{\mathcal{A}}(\mathbf{a})\right)=\sum_{n=0}^{\infty} P_{n}\left(\left(m \otimes \mathbf{1}_{X}\right) \mathbf{a}\right)=f\left(\left(m \otimes \mathbf{1}_{X}\right) \mathbf{a}\right) .
$$

This proves (11), which implies (12) and completes the proof.

Our next lemma yields a simplified proof of a similar result in [8].

Lemma 5. Let $\mathcal{A}$ denote a unital Banach algebra, $\gamma$ a uniform crossnorm, $X$ a Banach space with the bounded projection property and bounded set of projections $\left(T_{\alpha}\right)_{\alpha}$ converging to the identity and let $\sigma^{*}$ be a spectral system. Then given a commutative in $\mathcal{A} \widehat{\otimes}_{\gamma} X$ and $c>1$ there exists $\delta>0$ and for every $\varepsilon>0$ a direct sum decomposition $X=X_{1}(\varepsilon) \oplus X_{2}(\varepsilon)$ with $X_{1}(\varepsilon)$ finite-dimensional, a decomposition of $\mathbf{a}, \mathbf{a}=\mathbf{a}_{1}(\varepsilon)+\mathbf{a}_{2}(\varepsilon)$, and open sets $U_{1}(\varepsilon) \subset X_{1}(\varepsilon)$ and $U_{2}(\varepsilon) \subset X_{2}(\varepsilon)$ such that the following hold for all $\varepsilon>0$ : 
1. $\mathbf{a}_{i}(\varepsilon) \in \mathcal{A}_{\mathbf{a}}, i=1,2$,

2. $U_{2}(\varepsilon)=\varepsilon B \cap X_{2}(\varepsilon)$ where $B=\{x \in X:\|x\|<1\}$,

3. $\sigma^{*}\left(\mathbf{a}_{1}(\varepsilon)\right) \subset U_{1}(\varepsilon)$ and $c\left\|\mathbf{a}_{2}(\varepsilon)\right\|<\varepsilon$,

4. $\sigma^{*}(\mathbf{a})+\varepsilon B \subset U_{1}(\varepsilon) \oplus U_{2}(\varepsilon) \subset \sigma^{*}(\mathbf{a})+\varepsilon \delta B$.

Proof. Let $d=\sup _{\alpha}\left\|T_{\alpha}\right\|$ and let $T^{\alpha}:=I_{X}-T_{\alpha}$. Then $X=T_{\alpha}(X) \oplus$ $T^{\alpha}(X)$ for all $\alpha$ where both $T_{\alpha}(X)$ and $T^{\alpha}(X)$ are given the induced norm from $X$. Fix $\varepsilon>0$. By Proposition $8,\left(T^{\alpha}\right)_{\mathcal{A}}(\mathbf{a}) \rightarrow 0$ as $\alpha \rightarrow \infty$ and we may choose $\beta:=\alpha_{\varepsilon}$ such that $c\left\|\left(T^{\beta}\right)_{\mathcal{A}}(\mathbf{a})\right\|<\varepsilon$. Let

$$
\mathbf{a}_{2}:=\mathbf{a}_{2}(\varepsilon):=\left(T^{\beta}\right)_{\mathcal{A}}(\mathbf{a})=\left(\mathbf{e} \otimes T^{\beta}\right) \mathbf{a}
$$

and let $\mathbf{a}_{1}:=\mathbf{a}_{1}(\varepsilon):=\mathbf{a}-\mathbf{a}_{2}=\left(T_{\beta}\right)_{\mathcal{A}}(\mathbf{a})=\left(\mathbf{e} \otimes T_{\beta}\right) \mathbf{a}$. By Proposition 9 , $\mathbf{a}_{i} \in \mathcal{A}_{\mathbf{a}}$, and by Proposition $11, \sigma^{*}\left(\mathbf{a}_{i}\right) \subset X_{i}(\varepsilon)$ for $i=1,2$.

Let

$$
U_{1}(\varepsilon)=\sigma^{*}\left(\mathbf{a}_{1}\right)+2 \varepsilon d\left(B \cap T_{\beta}(X)\right), \quad U_{2}(\varepsilon)=2 \varepsilon(1+d)\left(B \cap T^{\beta}(X)\right) .
$$

If $x \in \sigma^{*}(\mathbf{a})$ then $x=T_{\beta}(x)+T^{\beta}(x)$, where $T_{\beta}(x) \in \sigma^{*}\left(\mathbf{a}_{1}\right)$, and by Proposition $3,\left\|T^{\beta}(x)\right\|<\varepsilon$. Hence

$$
\begin{aligned}
\sigma^{*}(\mathbf{a})+\varepsilon B & \subset \sigma^{*}\left(\mathbf{a}_{1}\right)+2 \varepsilon B \\
& \subset \sigma^{*}\left(\mathbf{a}_{1}\right)+2 \varepsilon d\left(B \cap T_{\beta}(X)\right)+2 \varepsilon(1+d)\left(B \cap T^{\beta}(X)\right) \\
& =U_{1}(\varepsilon) \oplus U_{2}(\varepsilon) \subset \sigma^{*}(\mathbf{a})+\varepsilon(3+4 d) B .
\end{aligned}
$$

On taking $\delta=3+4 d$ the proof is complete.

If $K$ is a compact subset of a Banach space we let $\mathcal{H}(K)$ denote the space of holomorphic germs on $K$. The compact-open topology $\tau_{0}$ on $\mathcal{H}(K)$ is defined to be the inductive limit of $\left(\mathcal{H}(U), \tau_{0}\right)$ where $U$ ranges over the open neighbourhoods of $K$. The $\tau_{\mathrm{b}}$ topology on $\mathcal{H}(K)$ is defined as the inductive limit topology derived from the spaces $\left(\mathcal{H}_{\mathrm{b}}(U), \tau_{\mathrm{b}}\right), U$ ranging over the open subsets of $X$ containing $K$. We require the following well known fact about holomorphic functions:

$$
H_{\mathrm{b}}(U \times V) \equiv H(U) \widehat{\otimes}_{\pi} H_{\mathrm{b}}(V)
$$

when $U$ is an open subset of a finite-dimensional space and $V$ is an open subset of a Banach space.

By Lemma 5 we can choose a null sequence $\left(\varepsilon_{n}\right)_{n=1}^{\infty}$ of positive numbers such that $\left(U_{1}\left(\varepsilon_{n}\right) \oplus U_{2}\left(\varepsilon_{n}\right)\right)_{n=1}^{\infty}$ is a decreasing basic open neighbourhood system for $\sigma^{*}(\mathbf{a})$. This implies (see $[4,5]$ )

$$
\left(H\left(\sigma^{*}(\mathbf{a})\right), \tau\right)=\lim _{n}\left(H_{\mathrm{b}}\left(U_{1}\left(\varepsilon_{n}\right) \oplus U_{2}\left(\varepsilon_{n}\right)\right), \tau\right)
$$

where $\tau=\tau_{0}$ or $\tau_{\mathrm{b}}$.

To extend the functional calculus in Lemma 4 we need a further condition on $\sigma^{*}$. The existence of the set $\mathcal{S}^{*}\left(\mathcal{A}_{\mathbf{a}}\right)$ in the following definition is proved in Proposition 2. 
Definition 6. A spectral system $\sigma^{*}$ admits a unique finite $\mathcal{A}$-holomorphic functional calculus if for all $\mathbf{a}:=\left(a_{1}, \ldots, a_{n}\right) \in \mathfrak{C}_{0}(\mathcal{A})$ and every open set $U$ containing $\sigma^{*}(\mathbf{a})$ there exists a unique $\tau_{0}$-continuous algebra homomorphism $\theta_{\mathbf{a}}: \mathcal{H}(U) \rightarrow \mathcal{A}$ such that

$$
\theta_{\mathbf{a}}\left(x^{\prime}\right)=x_{\mathcal{A}}^{\prime}(\mathbf{a})
$$

for all $x^{\prime} \in\left(\mathbb{C}^{n}\right)^{\prime}$ and

$$
m\left(\theta_{\mathbf{a}}(f)\right)=f\left(\left(m \otimes \mathbf{1}_{\mathbb{C}^{n}}\right) \mathbf{a}\right)
$$

for all $f \in \mathcal{H}(U)=\mathcal{H}_{\mathrm{b}}(U)$ and all $m \in \mathcal{S}^{*}\left(\mathcal{A}_{\mathbf{a}}\right)$.

Proposition 12. Let $\mathcal{A}$ denote a Banach algebra with identity e, $X$ a Banach space which is a complemented subspace of a Banach space with the bounded projection property, and let $\mathbf{a}$ denote a commutative element in $\mathcal{A} \widehat{\otimes}_{\gamma}$ $X$ where $\gamma$ is a uniform cross-norm. If $X$ is $(\mathbf{a}, \gamma)$ adaptable with constant $c^{*}$ satisfying (6) and $\sigma^{*}$ is a spectral system which admits a unique finite $\mathcal{A}$ holomorphic functional calculus, then for every open set $U$ containing $\sigma^{*}(\mathcal{A})$ there exists a unique $\tau_{\mathrm{b}}$-continuous algebra homomorphism $\theta_{U}:=\theta_{\mathbf{a}, U}: f \in$ $\mathcal{H}_{\mathrm{b}}(U) \mapsto f_{\mathcal{A}}(\mathbf{a}) \in \mathcal{A}$ which is $\tau_{0}$-continuous on bounded sets such that

$$
\theta_{U}\left(x^{\prime}\right)=\left(x^{\prime}\right)_{\mathcal{A}}(\mathbf{a})
$$

for all $x^{\prime} \in X^{\prime}$ and

$$
m\left(\theta_{U}(f)\right)=f\left(\left(m \otimes \mathbf{1}_{X}\right) \mathbf{a}\right)
$$

for all $f \in \mathcal{H}_{\mathrm{b}}(U)$ and all $m \in \mathcal{S}^{*}\left(\mathcal{A}_{\mathbf{a}}\right)$.

Proof. It suffices to establish the result for a fundamental system of neighbourhoods of $\sigma^{*}(\mathbf{a})$. We first suppose that $X$ has the bounded projection property. Fix $\varepsilon>0$. We use our previous notation: $U:=U_{1} \oplus U_{2}$ where $U_{i} \subset X_{i}(\varepsilon)=: X_{i}, U_{2}:=\varepsilon B \cap X_{2}, \mathbf{a}_{i}:=\mathbf{a}_{i}(\varepsilon), i=1,2$, and $d=$ $\sup _{\alpha}\left\|T_{\alpha}\right\|$ are defined as in Lemma 5. In this setting each $f \in \mathcal{H}_{\mathrm{b}}\left(U_{1} \oplus U_{2}\right)$ has an expansion $f=\sum_{n=1}^{\infty} \alpha_{n} f_{n} \otimes g_{n}$ where $\left(\alpha_{n}\right)_{n=1}^{\infty} \subset l_{1},\left(f_{n}\right)_{n=1}^{\infty}$ is a bounded subset of $\left(H\left(U_{1}\right), \tau_{0}\right),\left(g_{n}\right)_{n=1}^{\infty}$ is a bounded subset of $\left(H_{\mathrm{b}}\left(U_{2}\right), \tau_{\mathrm{b}}\right)$ and $\sum_{n=0}^{\infty}\left|\alpha_{n}\right| \cdot\left\|f_{n}\right\|_{K} \cdot\left\|g_{n}\right\|_{B_{\delta}}<\infty$ for every compact subset $K \subset U_{1}$ and all $\delta<\varepsilon$.

Since $\mathcal{A}_{\mathbf{a}_{2}} \subset \mathcal{A}_{\mathbf{a}}$ by Proposition 9 and $X_{2}$ is a complemented subspace of $X$ it is easily verified that $X_{2}$ is $\left(\mathbf{a}_{2}, \gamma\right)$ adaptable with constant $c:=$ $c^{*}(1+d)$, independent of $\varepsilon$, satisfying (6). By Lemma $5, c\left\|\mathbf{a}_{2}\right\|<\varepsilon$.

Let $\theta_{\mathbf{a}_{2}}: \mathcal{H}\left(U_{2}\right) \rightarrow \mathcal{A}_{\mathbf{a}}$ denote the homomorphism defined in Lemma 4 and let $\theta_{\mathbf{a}_{1}}: \mathcal{H}\left(U_{1}\right) \rightarrow \mathcal{A}$ denote the unique homomorphism given by the finite-dimensional functional calculus. If $\theta_{\mathbf{a}}:=\theta_{\mathbf{a}_{1}} \otimes \theta_{\mathbf{a}_{2}}: \mathcal{H}_{\mathrm{b}}\left(U_{1} \oplus U_{2}\right) \rightarrow \mathcal{A}$ let

$$
\theta_{\mathbf{a}}\left(\sum_{n=1}^{\infty} \alpha_{n} f_{n} \otimes g_{n}\right)=\sum_{n=0}^{\infty} \alpha_{n} \theta_{\mathbf{a}_{1}}\left(f_{n}\right) \cdot \theta_{\mathbf{a}_{2}}\left(g_{n}\right) .
$$


The mapping $\theta_{\mathbf{a}}$ is an algebra homomorphism which is $\tau_{\mathrm{b}}$-continuous on $\mathcal{H}_{\mathrm{b}}(U)$ and $\tau_{0}$-continuous on the bounded subsets of $\mathcal{H}_{\mathrm{b}}(U)$. The proof of Lemma 21 in [8] shows that $\theta_{\mathbf{a}}$ has all the required properties and the proof of Theorem 18 in [8] shows how the result can be extended to complemented subspaces. This completes the proof.

Under the same hypotheses we have the following corollary.

COROLlary 1 . There exists a unique $\tau_{0}$-continuous algebra homomorphism $\theta:=\theta_{\mathbf{a}}: f \in \mathcal{H}\left(\sigma^{*}(\mathbf{a})\right) \rightarrow f_{\mathcal{A}}(\mathbf{a}) \in \mathcal{A}$ with the following properties:

$$
\theta_{\mathbf{a}}\left(x^{\prime}\right)=x_{\mathcal{A}}^{\prime}(\mathbf{a})
$$

for all $x^{\prime} \in X^{\prime}$,

$$
m\left(\theta_{\mathbf{a}}(f)\right)=f\left(\left(m \otimes \mathbf{1}_{X}\right) \mathbf{a}\right) \quad \text { and } \quad \sigma^{*}\left(\theta_{\mathbf{a}}(f)\right)=f\left(\sigma^{*}(\mathbf{a})\right)
$$

for all $f \in \mathcal{H}\left(\sigma^{*}(\mathbf{a})\right)$ and all $m \in \mathcal{S}^{*}\left(\mathcal{A}_{\mathbf{a}}\right)$.

Proof. If $U$ and $V$ are open subsets of $X$ and $V \subset U$ we let $R_{U, V}(f)=\left.f\right|_{V}$ denote the restriction operator from $\mathcal{H}(U)$ to $\mathcal{H}(V)$. The mapping $R_{U, V}$ is an algebra homomorphism. If $\sigma^{*}(\mathbf{a}) \subset V \subset U$ then, by uniqueness, we have $\theta_{U} \circ R_{U, V}=\theta_{V}$. The definition of inductive limits implies that we obtain the required homomorphism and that it is unique. The continuity property follows, as in [8], from a result of Mujica [12]. This completes the proof.

ExAmple 3. Let $\mathcal{A}$ denote a commutative unital Banach algebra. For $\left(a_{i}\right)_{i=1}^{n} \subset \mathcal{A}$ we let $\sigma\left(\left(a_{i}\right)_{i=1}^{n}\right)=\left\{\left(m\left(a_{i}\right)\right)_{i=1}^{n}: m \in \mathcal{S}(\mathcal{A})\right\}$. This is the classical joint spectrum based on the commutative Gelfand theory which satisfies the hypothesis in Definition 1 and hence is a spectral system. Results of Zame [21] and Putinar [15] show that $\sigma$ admits a unique finite $\mathcal{A}$-holomorphic functional calculus. An infinite-dimensional holomorphic functional calculus for this spectrum was obtained in [8] and many of the techniques developed for that particular case were used in this article. As it was first developed in an infinite-dimensional tensor product setting by Waelbroeck [20] we refer to it as the Gelfand-Waelbroeck spectrum and denote it by $\sigma_{\mathrm{W}}$. If $\mathbf{a} \in \mathcal{A} \widehat{\otimes}_{\gamma} X$ then

$$
\sigma_{\mathrm{W}}(\mathbf{a})=\{(m \otimes \mathbf{1})(\mathbf{a}): m \in \mathcal{S}(\mathcal{A})\} .
$$

EXAMPLE 4. The most important joint spectrum for a finite number of commuting elements in the non-commutative Banach algebra $\mathcal{B}(Y)$ is the joint spectrum $\sigma_{\mathrm{T}}$ introduced by J. L. Taylor $[18,19]$. Talyor showed that $\sigma_{\mathrm{T}}$ satisfies the conditions in Definition 1 and hence $\sigma_{\mathrm{T}}$ is a spectral system. Putinar [14] established that $\sigma_{\mathrm{T}}$ admits a unique finite $\mathcal{B}(Y)$-holomorphic functional calculus for any Banach space $Y$. If $\mathbf{a}:=\left(a_{i}\right)_{i=1}^{n} \subset \mathcal{B}(Y)$ then, by $[18,19], \theta_{\mathbf{a}}(f) \subset \mathbf{a}^{\prime \prime}$, the double commutant of $\left(a_{i}\right)_{i=1}^{n}$ in $\mathcal{B}(Y)$, for any $f \in \mathcal{H}\left(\sigma_{\mathrm{T}}(\mathbf{a})\right)$. If the conditions on $\mathbf{a} \in \mathcal{B}(Y) \widehat{\otimes}_{\gamma} X, \gamma$ and $X$ in Proposition 12 hold then, in view of Lemma 4 and (15), the mapping $\theta_{\mathbf{a}}$ in Proposition 12 
maps $\mathcal{H}(U), U$ a neighbourhood of $\sigma_{\mathrm{T}}(\mathbf{a})$, into $\mathcal{A}_{\mathbf{a}}^{\prime \prime}$, the double commutant of $\mathcal{A}_{\mathbf{a}}$ in $\mathcal{B}(Y)$.

ExAmple 5. If $\mathcal{A}$ is a unital Banach algebra and $a \in \mathcal{A}$ let $L_{a}$ denote the operation of multiplication by $a$. Then $L_{a} \in \mathcal{B}(\mathcal{A})$ and if $\mathbf{a}=\left(a_{i}\right)_{i=1}^{n} \subset \mathfrak{C}_{0}(\mathcal{A})$ then $L_{\mathbf{a}}:=\left(L_{a_{i}}\right)_{i=1}^{n} \in \mathfrak{C}_{0}(\mathcal{B}(\mathcal{A}))$. The Taylor split spectrum of a, $\sigma_{\mathrm{T} \text {,split }}(\mathbf{a})$, is defined to be $\sigma_{\mathrm{T}}\left(L_{\mathbf{a}}\right)$. If $\mathcal{A}$ is commutative then, by a remark in the proof of Corollary 5.21 in [2], $\sigma_{\mathrm{W}}(\mathbf{a})=\sigma_{\mathrm{T}}\left(L_{\mathbf{a}}\right)$ and hence the Taylor split spectrum generalizes the Gelfand-Waelbroeck spectrum. The Taylor split spectrum is a spectral system and not necessarily [13] the same as the split spectrum when $\mathcal{A}=\mathcal{B}(Y)$.

If $\mathbf{a}$ and $\mathbf{b}$ are commutative elements in $\mathcal{B}(Y) \widehat{\otimes}_{\gamma} X$ where $X$ and $Y$ are Banach spaces and $T \in \mathcal{B}(Y)$ then we say that $T$ intertwines $\mathbf{a}$ and $\mathbf{b}$ if $\left(x^{\prime}\right)_{\mathcal{A}}(\mathbf{a}) \circ T=T \circ\left(x^{\prime}\right)_{\mathcal{A}}(\mathbf{b})$ for all $x^{\prime} \in X^{\prime}$. Our final proposition extends Theorem 4.5 in [19] from finite-dimensional spaces to Banach spaces with the bounded projection property. In the proof $\left(T_{\alpha}\right)_{\alpha}$ will denote a bounded set of finite rank projections which converges uniformly to the identity $\mathbf{1}_{X}$.

Proposition 13. Let $X$ and $Y$ be Banach spaces and suppose $X$ has the bounded projection property. Let $\mathbf{a}$ and $\mathbf{b}$ denote commutative elements in $\mathcal{B}(Y) \widehat{\otimes}_{\gamma} X$ where $\gamma$ is a uniform cross-norm such that $X$ is both $(\mathbf{a}, \gamma)$ and $(\mathbf{b}, \gamma)$ adaptable. If $T \in \mathcal{B}(Y)$ intertwines $\mathbf{a}$ and $\mathbf{b}$ and $f \in \mathcal{H}(U)$ where $U$ is an open subset of $X$ containing $\sigma_{\mathrm{T}}(\mathbf{a}) \cup \sigma_{\mathrm{T}}(\mathbf{b})$, then $T$ intertwines $f_{\mathbf{A}}(\mathbf{a})$ and $f_{\mathbf{A}}(\mathbf{b})$.

Proof. By (5), T intertwines $\left(\phi^{n}\right)_{\mathcal{A}}(\mathbf{a})$ and $\left(\phi^{n}\right)_{\mathcal{A}}(\mathbf{b})$ for all $\phi \in X^{\prime}$ and, by the bounded projection property, $T$ intertwines $P_{\mathcal{A}}(\mathbf{a})$ and $P_{\mathcal{A}}(\mathbf{b})$ for all $P \in \mathcal{P}(X)$. If $S \in \mathcal{B}(X)$ then $\left(x^{\prime}\right)_{\mathcal{A}}\left(S_{\mathcal{A}}(\mathbf{a})\right)=\left(x^{\prime} \circ S\right)_{\mathcal{A}}(\mathbf{a})$ for all $x^{\prime} \in X^{\prime}$. Since $x^{\prime} \circ S \in X^{\prime}$ this implies that $T$ intertwines $S_{\mathcal{A}}(\mathbf{a})$ and $S_{\mathcal{A}}(\mathbf{b})$. Combining these two cases we see that $T$ intertwines $(P \circ S)_{\mathcal{A}}(\mathbf{a})$ and $(P \circ S)_{\mathcal{A}}(\mathbf{b})$.

We now use (16) and keep the notation used in that equation. Since $\theta_{\mathbf{a}_{2}}\left(g_{n}\right)=\sum_{n=0}^{\infty}\left(P_{n} \circ T^{\alpha}\right)_{\mathcal{A}}(\mathbf{a})$ and $\theta_{\mathbf{b}_{2}}\left(g_{n}\right)=\sum_{n=0}^{\infty}\left(P_{n} \circ T^{\alpha}\right)_{\mathcal{A}}(\mathbf{b})$ for some $\alpha$ where $P_{n} \in \mathcal{P}\left({ }^{n} X\right)$ for all $n$ and $T^{\alpha}=I_{X}-T_{\alpha}$ the above shows that $T$ intertwines $\theta_{\mathbf{a}_{2}}\left(g_{n}\right)$ and $\theta_{\mathbf{b}_{2}}\left(g_{n}\right)$. Now $T$ intertwines $\mathbf{a}_{1}=\left(T_{\alpha}\right)_{\mathcal{A}}(\mathbf{a})$ and $\mathbf{b}_{1}=\left(T_{\alpha}\right)_{\mathcal{A}}(\mathbf{b})$ and the finite-dimensional intertwining result shows that $T$ intertwines $\theta_{\mathbf{a}_{1}}\left(f_{n}\right)$ and $\theta_{\mathbf{b}_{1}}\left(f_{n}\right)$. By continuity, linearity and (16) it follows that $T$ intertwines $f_{\mathcal{A}}(\mathbf{a})$ and $f_{\mathcal{A}}(\mathbf{b})$. This completes the proof.

\section{References}

[1] G. Botelho, Type, cotype and generalized Rademacher functions, Rocky Mountain J. Math. 28 (1998), 1227-1250. 
[2] R. E. Curto, Applications of several complex variables to multiparameter spectral theory, in: Surveys of Some Recent Trends in Operator Theory, Vol. II, J. B. Conway and B. B. Morrel (eds.), Longman Sci. Tech., Harlow, 1988, 25-90.

[3] A. Defant and K. Floret, Tensor Norms and Operator Ideals, North-Holland Math. Stud. 176, North-Holland, Amsterdam, 1993.

[4] S. Dineen, Complex Analysis on Infinite Dimensional Spaces, Springer Monogr. Math., Springer, London, 1999.

[5] - Spectral theory, tensor products and infinite dimensional holomorphy, J. Korean Math. Soc. 41 (2004), 193-207.

[6] S. Dineen, R. E. Harte and C. Taylor, Spectra of tensor product elements I: Basic theory, Math. Proc. Roy. Irish Acad. 101A (2001), 177-196.

[7] - - - - Spectra of tensor product elements II: Polynomial extensions, ibid. 101A (2001), 197-220.

[8] - - - - , Spectra of tensor product elements III: Holomorphic properties, ibid. 103A (2003), 61-92.

[9] J. Eschmeier and M. Putinar, Spectral Decompositions and Analytic Sheaves, London Math. Soc. Monogr. 10, Oxford Univ. Press, New York, 1996.

[10] R. E. Harte, The spectral mapping theorem in many variables, in: Seminar on Uniform Algebras, Dept. of Math., Univ. of Aberdeen, Aberdeen, 1973, 59-63.

[11] -, Invertibility and Singularity for Bounded Linear Operators, Monogr. Textbooks Pure Appl. Math. 109, Dekker, New York, 1988.

[12] J. Mujica, A Banach-Dieudonné theorem for germs of holomorphic functions, J. Funct. Anal. 57 (1984), 31-48.

[13] V. Müller, The splitting spectrum differs from the Taylor spectrum, Studia Math. 123 (1997), 291-294.

[14] M. Putinar, Uniqueness in Taylor's functional calculus, Proc. Amer. Math. Soc. 89 (1983), 647-650.

[15] -, Functional calculus and the Gelfand transformation, Studia Math. 79 (1984), 83-86.

[16] R. A. Ryan, Introduction to Tensor Products of Banach Spaces, Springer Monogr. Math., Springer, London, 2002.

[17] Z. Słodkowski and W. Żelazko, On joint spectra of commuting families of operators, Studia Math. 50 (1974), 127-148.

[18] J. L. Taylor, A joint spectrum for several commuting elements, J. Funct. Anal. 6 (1970), 172-191.

[19] -, The analytic-functional calculus for several commuting operators, Acta Math. 125 (1970), 1-38.

[20] L. Waelbroeck, Topological Vector Spaces and Algebras, Lecture Notes in Math. 230, Springer, Belin, 1973.

[21] W. R. Zame, Existence and uniqueness of functional calculus homomorphisms, Bull. Amer. Math. Soc. 82 (1976), 123-125.

[22] W. Żelazko, On axiomatic spectra I, Studia Math. 64 (1979), 249-261.

University College Dublin

Belfield, Dublin 4, Ireland

E-mail: sdineen@maths.ucd.ie
School of Mathematics Trinity College Dublin

Dublin 2, Ireland E-mail: rharte@maths.tcd.ie 